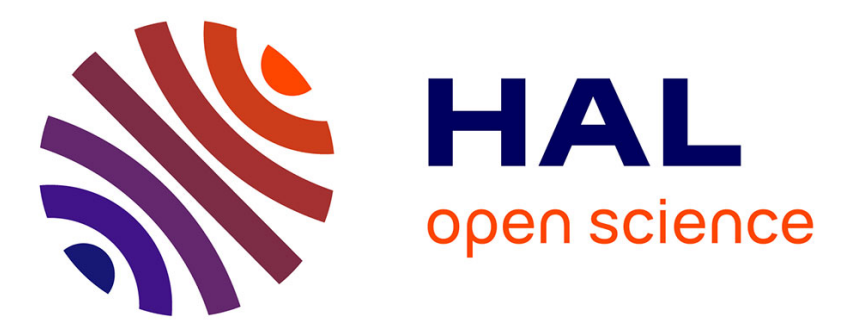

\title{
On depth-averaged analytic solutions for free-surface granular flows impacting rigid walls down inclines \\ Thierry Faug
}

\section{To cite this version:}

Thierry Faug. On depth-averaged analytic solutions for free-surface granular flows impacting rigid walls down inclines. Physical Review E : Statistical, Nonlinear, and Soft Matter Physics, 2015, 92 (6), 14 p. 10.1103/PhysRevE.92.062310 . hal-01275252

\section{HAL Id: hal-01275252 \\ https://hal.science/hal-01275252}

Submitted on 17 Feb 2016

HAL is a multi-disciplinary open access archive for the deposit and dissemination of scientific research documents, whether they are published or not. The documents may come from teaching and research institutions in France or abroad, or from public or private research centers.
L'archive ouverte pluridisciplinaire HAL, est destinée au dépôt et à la diffusion de documents scientifiques de niveau recherche, publiés ou non, émanant des établissements d'enseignement et de recherche français ou étrangers, des laboratoires publics ou privés. 


\title{
On depth-averaged analytic solutions for free-surface granular flows impacting rigid walls down inclines
}

\author{
Thierry Faug* \\ Irstea, Univ. Grenoble-Alpes, UR ETGR, \\ F-38402 St Martin d'Heres, France ${ }^{\dagger}$
}

(Dated: December 4, 2015)

\begin{abstract}
In the present paper, flows of granular materials impacting wall-like obstacles down inclines are described by depth-averaged analytic solutions. Particular attention is paid to extending the existing depth-averaged equations initially developed for frictionless and incompressible fluids down a horizontal plane. The effects of the gravitational acceleration along the slope, and of the retarding acceleration caused by friction as well, are systematically taken into account. The analytic solutions are then used to revisit existing data on rigid walls impacted by granular flows. This approach allows establishing a complete phase-diagram for granular flow-wall interaction.
\end{abstract}

\footnotetext{
* thierry.faug@irstea.fr

$\dagger$ School of Civil Engineering, University of Sydney, NSW 2006, Australia
} 


\section{INTRODUCTION}

The influence of obstacles on the propagation of granular flows is today a booming research area. Flow-obstacle interactions in granular flows have recently drawn attractions from different disciplines in science and technology because the flows past obstacles are locally complex and highly non-linear. Flow regimes can change from fast to slow states, as well as from diverging to converging states, and vice versa.

There are for instance several studies in geophysics which used laboratory tests on granular flows to mimic full-scale mitigation structures against avalanches, in order to analyse some complex problems yet to be solved: the runup on avalanche catching dams [1-5], the runout shortening caused by catching dams or dissipative structures such as retarding mounds [6-11], the deflection induced by a snow shed [12], a catching dam [13] or a deflecting dam [14], and the effect of a complex protection system including mounds and dams [15]. Other small-scale studies used numerical simulations based on depth-averaged models [16], discrete element method [17] or full dimensional models coupled with depth-averaged models [18] to tackle the influence of retarding mounds, wedges or walls on the flow geometry. Many authors have recently combined small-scale numerical simulations and laboratory tests [19-24]. More rarely, some controlled experiments on flows of geomaterials - such as snow-interacting with obstacles were carried out on large-scale flumes to investigate the effect of snow sheds [25], retarding mounds [26] or deflecting dams [27] but those experiments remain costly.

The overarching aim of the present paper is to pay attention to rigid walls spanning the whole width of the incoming granular flow. The numerical simulations based on discrete element method [17, 23, 28-31] or the full dimensional continuum models, as recently developed by Domnik and Pudasaini [32], are important and necessary contributions to the topic. In the present paper, various analytic solutions are proposed for describing the main kinematics of the granular patterns formed when flows of granular materials hit walls down inclines. A depth-averaged approach, which ignores the details of the discrete nature of the flowing material, is proposed. The particular behaviour of flowing grains is considered through choosing relevant values of the effective parameters that characterize the assembly of grains.

The depth-averaged analytic solutions proposed in the present study systematically take 
into account the effects of both the potential energy caused by gravity and the energy dissipated by friction, as recently done for the kinematics of granular flows without any obstacle by Pudasaini and Domnik [33]. Previous studies on small-scale granular flows past rigid walls are revisited and cross-compared in order to draw a complete phase-diagram. The latter is helpful for evidencing the conditions needed to form each of the various granular patterns induced by flow-wall interaction.

The present paper is organized as follows. Section II describes the general depth-averaged analytic solutions for bulky frictional granular flows impacting rigid walls down inclines. In the first place, the depth-averaged equations for flows without any obstacle are shortly presented (Sec. II A). Second, a general equation for the stationary granular jump is proposed (Sec. II B). Third, general equations for propagating granular jumps, namely granular bores, are given (Sec. II C). Fourth, the critical wall height below which the granular bore does no longer propagate upstream of an obstruction, and a large airborne jet is formed downstream of the wall, is tackled (Sec. IID). Section III revisites a great number of studies on various granular patterns resulting from flow-wall interaction, such as airborne granular jets, diffuse and strong granular jumps, and granular dead zones. Finally, a complete phasediagram governing the granular flow-wall interaction is prososed in Sec. IV, in line of the depth-averaged analytic solutions described in Sec. II.

\section{ANALYTIC SOLUTIONS FOR GRANULAR FLOWS IMPACTING WALLS}

\section{A. Depth-averaged equations for granular flows down inclines}

Gravity-driven free-surface flows of granular materials are well described by depthaveraged mass and momentum conservation equations. These equations were introduced in 1871 in order to describe the non-permanent water flows in application to river floods [34]. Then, the classical assumption of shallow water flows was applied to granular flows [35, 36] and motivated the mathematical modeling of natural hazards such as landslides [37], debris flows or mudflows [38, 39], rock avalanches [40] and snow avalanches [22, 41]. The key idea is to consider that the vertical length scale (the flow thickness $h$ ) is small with respect to the horizontal length scale $L$ along the slope. The assumption $\epsilon=h / L \ll 1$ is reasonable for many geophysical flows. It is then possible to depth-average the three-dimensional local 
conservation equations. The full derivation for granular avalanches can be found in [35, 36].

Mass [Eq.(1) below] and momentum [Eq.(2)] balances applied to an infinitesimal portion of fluid can quickly give the depth-averaged equations for a two-dimensional flow down a slope making an angle $\theta$ with the horizontal [42]:

$$
\begin{array}{r}
\frac{\partial h}{\partial t}+\frac{\partial h \bar{u}}{\partial x}=0 \\
\rho_{P} \phi\left(\frac{\partial h \bar{u}}{\partial t}+\beta \frac{\partial h \bar{u}^{2}}{\partial x}\right)=\rho_{P} \phi g h \cos \theta\left(\tan \theta-\mu_{b}-k \frac{\partial h}{\partial x}\right) .
\end{array}
$$

$h$ is the flow thickness and $\bar{u}=\frac{1}{h} \int_{0}^{h} u(z) d z$ is the depth-averaged velocity. $\rho_{P}$ is the particle density and $\phi$ is the volume fraction of the flowing granular material, which is constant (the fluid density is $\rho=\rho_{P} \phi$ ) as a direct result of $\epsilon \ll 1$. The $x$-coordinate corresponds to the direction of the main flow and $t$ is the time. $\beta$ is defined as the ratio between the depth-averaged value of the velocity square and the square of the depth-averaged velocity: $\beta=\overline{u^{2}} / \bar{u}^{2}$.

The forces at stake considered in Eq.(2) and balancing the inertial forces are: the flow weight $\left(\rho_{P} \phi g h \sin \theta\right)$, the friction force $\left(\tau_{b}=\mu_{b} \phi \rho_{P} g h \cos \theta\right.$, where $\mu_{b}$ is the basal effective friction coefficient) and the force related to the thickness gradient $\left(k \rho_{P} \phi g h \cos \theta \frac{\partial h}{\partial x}\right.$, where $k$ is the earth pressure coefficient relating the normal stresses $\sigma_{x x}$ and $\sigma_{z z}$ through $\sigma_{x x}=k \sigma_{z z}$ ).

The physical processes associated with the discrete nature of the granular medium — which forms the flowing layer - are not considered in detail. Relevant values should be given to the granular constitutive parameters $k, \beta$ and $\mu_{b}$, as it will be shortly discussed thereinafter.

The value of $k$ can be derived from soils mechanics concepts, as early suggested by Savage and Hutter [35]:

$$
k_{\text {pass } / \text { act }}=\frac{2\left(1 \pm \sqrt{1-\left(1+\tan ^{2} \delta\right) \cos ^{2} \varphi}\right)}{\cos ^{2} \varphi}-1,
$$

where $\delta$ is the bed friction angle, $\varphi$ is the internal friction angle and $k_{\text {pass } / a c t}=k_{\text {pass }}$ for a passive state $(\partial u / \partial x<0)$ or $k_{a c t}$ for an active state $(\partial u / \partial x>0)$. Eq.(3) with $\delta=\varphi$ yields $k_{\text {pass } / \text { act }}=\left(1+\sin ^{2} \varphi\right) /\left(1-\sin ^{2} \varphi\right)$. Past studies have shown that $k=1$ would be a reasonable assumption for sheared granular flows in the steady and uniform regime [43]. More recent studies have highlighted a slight anisotropy in normal stresses for the steady and uniform regime: $k=\sigma_{x x} / \sigma_{z z}=1.05$ [44]. It is likely that $k$ is influenced by the divergence 
of the flow [45], which may lead to values different from 1 when the free-surface is strongly non stationary and/or non uniform.

$\beta$ depends on the shape of the velocity profile. It is generally taken to be equal to 1 , which corresponds to the exact value for plug flows. It can be calculated provided an assumption on the shape of the velocity profile: $\beta=4 / 3$ for linear velocity profiles and $\beta=5 / 4$ for Bagnold velocity profiles.

$\mu_{b}$ can be taken as a constant [36] or more complex velocity-dependent friction laws can be considered. The granular constitutive friction laws are expressed through either frictionalcollisional concepts [46-49] or the dependence of $\mu_{b}$ on the inertial number, namely the $\mu(I)$-rheology described by MiDi [50] and Jop et al. [51].

The flow disturbed by the presence of an obstacle may have spatial variations $L$ whose scale is close to the typical flow thickness $h$. The shallow water flows assumption $(\epsilon=$ $h / L \ll 1$ ) is then no longer valid, as clearly evidenced by Pudasaini et al. [3], Pudasaini and Kröner [4], Faug et al. [10], Gray et al. [20], Johnson and Gray [52], etc.

This paper presents general depth-averaged equations in order to describe a steady granular flow down an incline and interacting with a rigid wall. The wall spans the whole width of the incident flow. Note that all the equations given in the following sections should apply to incoming flows (before impacting walls) in steady-state regime.

\section{B. Stationary granular jumps}

Granular jumps, namely large discontinuities in depth and velocity, are typically observed when a rapid granular flow hits a rigid wall. When overflow is possible downstream of the wall, a stationary granular jump (see Fig.1 in the current section) can be produced. When the obstruction prevents overflow downstream of the wall, the granular mass is entirely blocked and a granular bore propagates upstream from the obstruction (see Fig.3a in the next section).

\section{Full equation for steady jumps}

By analogy to hydraulic jumps [53], a theory can predict the change in depth in the granular jump [54-56]. A sketch of a stationary granular jump is given in Fig.1. $h_{i}, \bar{u}_{i}$ 


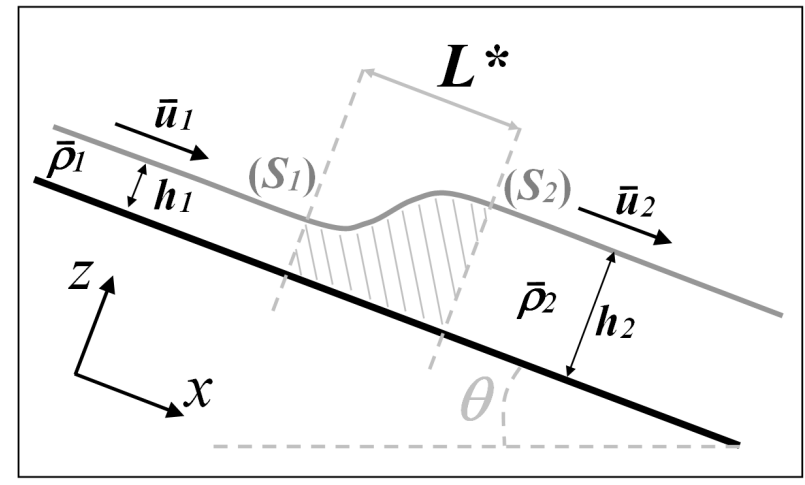

FIG. 1. Sketch of a stationary granular jump down an incline. The subscripts $i=1$ and 2 refer respectively to locations upstream (section $S_{1}$ ) and downstream (section $S_{2}$ ) of the jump.

and $\bar{\rho}_{i}$ are the thickness, the depth-averaged velocity and the depth-averaged density at the locations $i$, where the subscripts $i=1$ and 2 refer respectively to locations upstream and downstream of the jump region. By making use of the continuity equation $\left(\bar{\rho}_{1} \bar{u}_{1} h_{1}=\bar{\rho}_{2} \bar{u}_{2} h_{2}\right)$, the momentum equation applied to a control volume surrounding the jump yields:

$$
\bar{\rho}_{1} \bar{u}_{1} h_{1}\left(\beta_{2} \bar{u}_{2}-\beta_{1} \bar{u}_{1}\right)=P_{1}-P_{2}+W \sin \theta-F_{f} .
$$

$W$ is the weight of the granular material in the jump: $W=\frac{1}{2} \bar{K} g L_{*}\left(\bar{\rho}_{1} h_{1}+\bar{\rho}_{2} h_{2}\right)$, where $L_{*}$ is the length of the granular jump region (see Fig.1) and $\bar{K}$ is a coefficient accounting for the shape of the jump. $P_{1}$ and $P_{2}$ are the lateral pressure forces acting on sections $S_{1}$ and $S_{2}$ (see Fig.1): $P_{i}=\frac{1}{2} k_{i} \bar{\rho}_{i} g h_{i}^{2} \cos \theta$, where $k_{i}$ is the earth pressure coefficient (see discussion in Sec. II A). $F_{f}$ is the frictional force between locations 1 and 2 which can be expressed as $F_{f}=\mu_{e} W \cos \theta$, where $\mu_{e}$ is the effective coefficient of Coulomb friction within the jump volume, as early proposed by Savage [55] and recently revisited by Faug [57]. Some calculation allows deriving the relation between the Froude number $F r_{1}=\bar{u}_{1} / \sqrt{g h_{1} \cos \theta}$ of the incoming flow (defined at section $S_{1}$ in Fig.1), the jump depth ratio $h_{2} / h_{1}$ and the density variation $\bar{\rho}_{2} / \bar{\rho}_{1}$ across the jump:

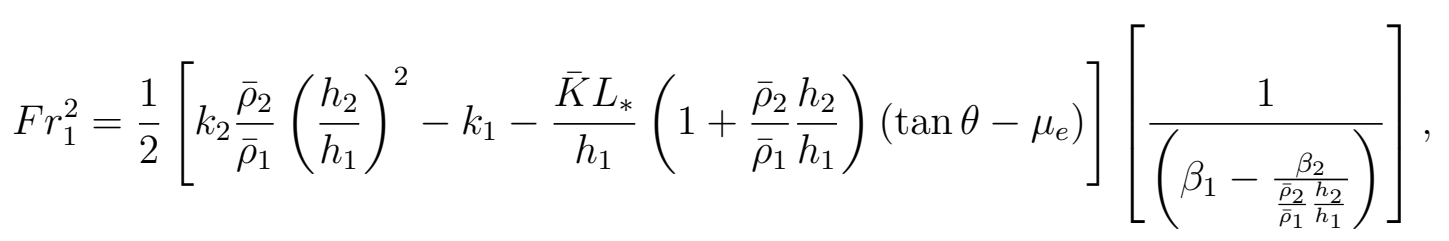

where various parameters, such as the earth pressure coefficients $k_{i}$ and the Boussinesq 
momentum coefficients $\beta_{i}$, can be estimated for granular flows (see Sec. II A). Apart from a recent study by Faug et al. [56] (see extended discussion in Sec.III A), past studies paid little attention to the other parameters appearing in Eq.(5): the jump shape parameter $\bar{K}$, the length of the jump relative to the thickness of the incoming flow $L_{*} / h_{1}$, and the difference between the tangent of the slope angle and the effective friction within the jump $\left(\tan \theta-\mu_{e}\right)$.

Fig.2a shows the jump depth ratio as a function of the Froude number of the incoming flow for different values of the density variation across the jump. The following values are used for the parameters in Eq.(5): $k_{i}=1, \beta_{i}=1, L_{*} / h_{1}=10$ (typical value measured in the experiments by Faug et al. [56]), $\bar{K}=1$ and $\tan \theta-\mu_{e}=0.11$ (which is for instance obtained from $\theta=30^{\circ}$ and $\mu_{e}=\tan \delta$ where $\delta=25^{\circ}$ ). The jump height increases with the Froude number but the increase rate is decreased when the density variation across the jump increases. Recent experiments by Faug et al. [56] showed that, at a given slope angle (which generally controls the Froude number), dilute flows produce jumps that are thinner than those produced by dense flows. This result is consistent with predictions shown in Fig.2a.

Incompressible jumps $\left(\bar{\rho}_{2} / \bar{\rho}_{1}=1\right)$ are considered in the following of the present section. How the relation between $h_{2} / h_{1}$ and $F r_{1}$ is influenced by $k_{i}, \beta_{i}, L_{*} / h_{1}, \bar{K}$ and $\tan \theta-\mu_{e}$ can be analysed then.

Fig.2b shows the jump depth ratio as a function of the Froude number for different values of $L_{*} / h_{1}$. Other parameters have the same values as in Fig.2a. The results show that the influence of $L_{*} / h_{1}$ is significant, in particular for low values of the Froude number of the incoming flow. Increasing $L_{*} / h_{1}$ generally produces an increase of the jump height relative to the incoming flow thickness, and the effect is enhanced at low Froude number.

Fig.2c depicts $h_{2} / h_{1}$ versus $F r_{1}$ for two values of $k_{2} . k_{1}$ is kept equal to 1 (isotropic flow conditions regarding normal stresses), which appears to be a reasonable assumption for the incoming flows in steady regime, as previously discussed in Sec. II A. Moreover, it can be verified that the influence of $k_{1}$ remains weak with respect to the influence of $k_{2}$. The jump height decreases while $k_{2}$ is increased from 1 up to 1.43, as shown in Fig.2c. The latter value for $k_{2}$ would correspond to an anisotropy in normal stresses under the following assumptions: (i) $\varphi=\delta=25^{\circ}$ and (ii) passive state downstream of the jump where $\partial u / \partial x<0$, which yields a sign + in Eq.(3). Other parameters have the same values as in Fig.2a with $\bar{\rho}_{2} / \bar{\rho}_{1}=1$.

Changes in $\beta_{i}$ from 1 to $5 / 4$ or $4 / 3$ (see discussion about those values in Sec.II) have very weak influence on the relation between $h_{2} / h_{1}$ and $F r_{1}$ (not shown here). Keeping $\beta_{i}=1$ is 

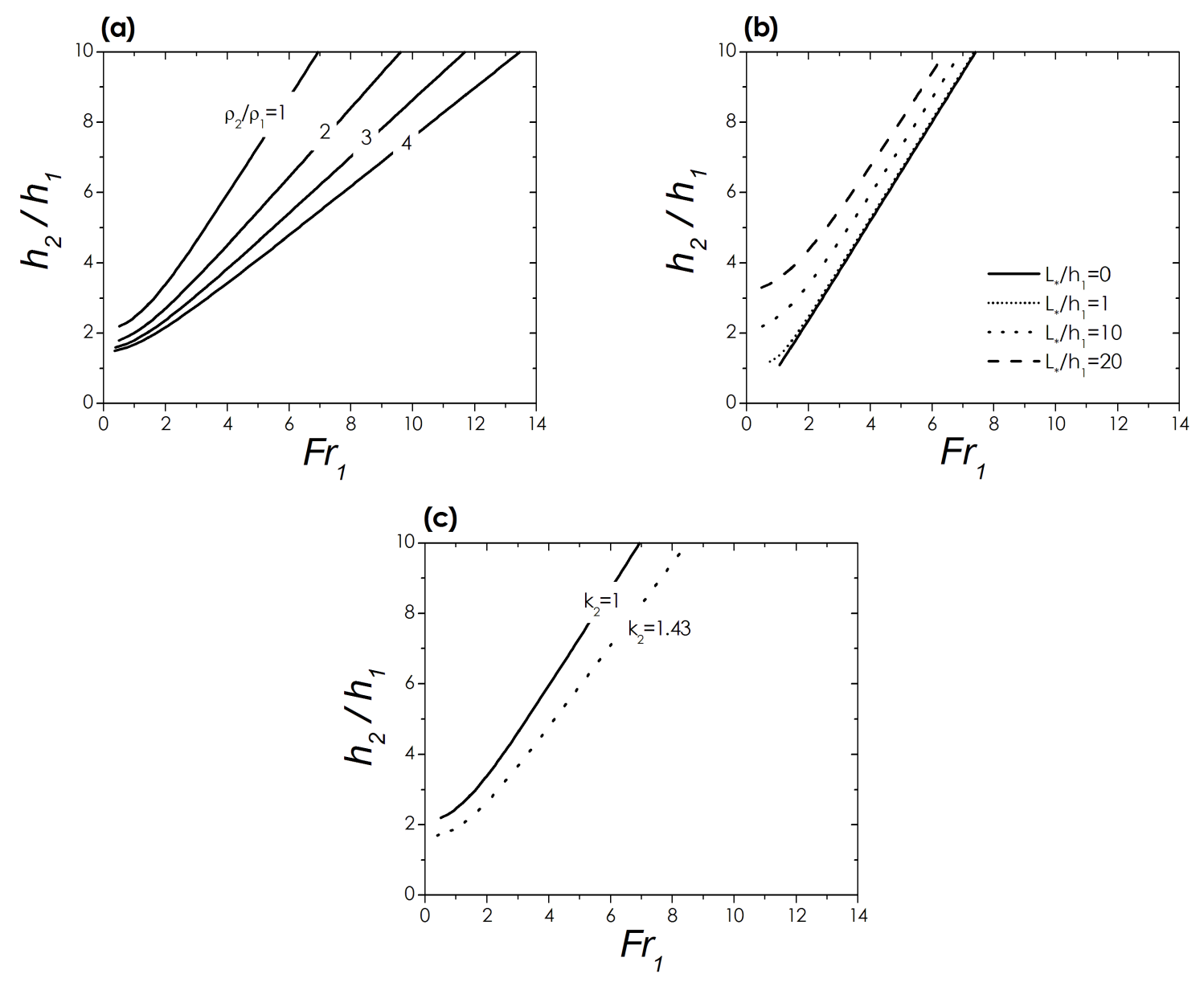

FIG. 2. Depth ratio $h_{2} / h_{1}$ versus the Froude number $F r_{1}$ from Eq.(5) for various values of $\bar{\rho}_{2} / \bar{\rho}_{1}$ (a), $L_{*} / h_{1}$ (b) and $k_{2}$ (c). The following values of the parameters needed for Eq.(5) are used for all graphs (unless one of these parameters is varied): $\bar{\rho}_{2} / \bar{\rho}_{1}=1, \beta_{i}=1, k_{i}=1, L_{*} / h_{1}=10$, $\tan \theta-\mu_{e}=0.11$, and $\bar{K}=1$.

then a reasonable assumption. The influence of $\bar{K}$ and $\tan \theta-\mu_{e}$ remain generally weak. A significant influence appears if the length of the jump relative to the thickness of the incoming flow, $L_{*} / h_{1}$, is large.

It can be concluded that the relation between $h_{2} / h_{1}, F r_{1}$ and $\bar{\rho}_{2} / \bar{\rho}_{1}$ is strongly influenced by the length of the jump, and, to a lesser extent, by a possible change in the earth pressure coefficient across the jump. 


\section{Simplified solution for incompressible steady jumps}

This section considers incompressible, or slightly compressible jumps, for which $\bar{\rho}_{2}=\bar{\rho}_{1}$. Such jumps are obtained if the incoming flow are not too dilute. Dilute flows produce compressible jumps for which the change in density across the jump cannot be ignored, as recently evidenced by [56]. Under the additional assumptions $k_{i}=k$ (no change in earth coefficient across the jump) and $\beta_{i}=\beta$ (no variation of velocity profiles across the jump), it is worth establishing that Eq.(5) can be written in the following form:

$$
\left(\frac{h_{2}}{h_{1}}\right)^{2}+\frac{h_{2}}{h_{1}}-\frac{2 \beta F r_{1}^{2}}{k-\bar{K} \frac{L_{*}}{h_{2}-h_{1}}\left(\tan \theta-\mu_{e}\right)}=0 .
$$

In hydraulics [53], it is generally assumed that the product $\bar{K} \frac{L_{*}}{\left(h_{2}-h_{1}\right)}$, which accounts for the geometry of the jump, can be expressed as a simple function of $F r_{1}$. Recent experiments on the shape of standing granular jumps [56] suggested that it is a reasonable assumption for granular flows too, provided that the jumps are incompressible or slightly compressiblemeaning that the density variation across the jump is nil or weak. The assumption that the Froude number of the incoming flow strongly controls the shape of the jump will be further discussed at section III A 1. Eq.(6) has the following solution:

$$
\frac{h_{2}}{h_{1}}=\frac{1}{2}\left[\left(1+\frac{8 \beta F r_{1}^{2}}{k-\bar{K} \frac{L_{*}}{h_{2}-h_{1}}\left(\tan \theta-\mu_{e}\right)}\right)^{1 / 2}-1\right] .
$$

For frictionless flows $\left(\mu_{e}=0\right)$ with no deviation from a purely hydrostatic pressure $(k=1)$ and uniform velocity profiles ( $\beta=1$ ), Eq.(7) leads to the traditional equation proposed for hydraulic jumps on steep slopes by Chow [53]:

$$
\frac{h_{2}}{h_{1}}=\frac{1}{2}\left[\left(1+\frac{8 F r_{1}^{2}}{1-\bar{K} \frac{L_{*}}{h_{2}-h_{1}} \tan \theta}\right)^{1 / 2}-1\right] .
$$

\section{Asymptotic solution for a granular flow over a small bump}

Eq.(7) can be used to describe a granular flow passing slowly over a bump of height $H_{0}$. The latter situation is similar to a very diffuse granular jump for which $h_{2}=H_{0}+h_{3}$ (where $h_{3}$ is the critical depth at the top of the bump), $h_{3}-h_{1}=\epsilon_{0}$ (where $\epsilon_{0} \sim 0$ ) and $\bar{K} \mapsto 1$. Eq.(7) yields: 


$$
\frac{H_{0}}{h_{1}}+\frac{\epsilon_{0}}{h_{1}}+1=\frac{1}{2}\left[\left(1+\frac{8 \beta F r_{1}^{2}}{k-\frac{L_{*}}{H_{0}+\epsilon_{0}}\left(\tan \theta-\mu_{e}\right)}\right)^{1 / 2}-1\right] .
$$

By considering $\epsilon_{0} \mapsto 0$ and $H_{0} / h_{1} \mapsto 0$ (small bump), one can derive a critical value $F r_{c}$ for the Froude number of the incoming flow:

$$
F r_{c}=\sqrt{\frac{1}{\beta}\left(k-\frac{L_{*}}{H_{0}}\left(\tan \theta-\mu_{e}\right)\right)} .
$$

The above equation suggests that the critical value of the Froude number-classically equal to 1 for horizontal water or air flows over a bump - would depend on $\beta, k,\left(\tan \theta-\mu_{e}\right)$, and $\frac{L_{*}}{H_{0}}$ in the case of frictional granular flows down inclines.

\section{Propagating jumps upstream of an obstruction}

The granular jump propagating upstream of an obstacle at speed $U$ (see sketch in Fig.3a), namely the granular bore, is for instance observed when a rapid granular flow hits a wall spanning the entire width of the flow $[3,58]$. Detailed features of that type of granular bore

have been investigated with the help of computations by Pudasaini and Kröner [4]. The granular bore can be also observed when the material flows past a change in slope from a high slope to a lower slope $[3,59]$. In geophysics, this situation is typical of some avalanches paths with a sharp change in slope which occurs at the transition from the flowing zone to the runout zone.

\section{Full equation for bores}

By choosing a reference frame travelling at the speed $U$ in the direction opposite to the incoming flow, mass and momentum conservation equations across the jump give (Fig.3a):

$$
\begin{array}{r}
\bar{\rho}_{1} h_{1}\left(\bar{u}_{1}+U\right)=\bar{\rho}_{2} h_{2} U, \\
\bar{\rho}_{2} h_{2} U^{2}-\bar{\rho}_{1} h_{1}\left(\bar{u}_{1}+U\right)^{2}=\frac{1}{2} k_{1} \bar{\rho}_{1} g h_{1}^{2} \cos \theta-\frac{1}{2} k_{2} \bar{\rho}_{2} g h_{2}^{2} \cos \theta+W \sin \theta-F_{f} .
\end{array}
$$

Vertical velocity profiles are assumed uniform for a sake of simplicity $(\beta=1)$. The weight of the jump $W$ and its effective friction $F_{f}$ with the bottom (and side-walls for 
confined flows) are determined by the equations proposed by Savage [55] and detailed in the previous section. A relation between the Froude number $F r_{1}=\bar{u}_{1} / \sqrt{g h_{1} \cos \theta}$, the depth ratio $h_{2} / h_{1}$ and the density ratio $\bar{\rho}_{2} / \bar{\rho}_{1}$ can be derived from Eqs.(11) and (12):

$$
F r_{1}^{2}=\frac{1}{2}\left[k_{2} \frac{\bar{\rho}_{2}}{\bar{\rho}_{1}}\left(\frac{h_{2}}{h_{1}}\right)^{2}-k_{1}-\frac{\bar{K} L_{*}}{h_{1}}\left(1+\frac{\bar{\rho}_{2}}{\bar{\rho}_{1}} \frac{h_{2}}{h_{1}}\right)\left(\tan \theta-\mu_{e}\right)\right]\left(1-\frac{1}{\frac{\bar{\rho}_{2}}{\bar{\rho}_{1}} \frac{h_{2}}{h_{1}}}\right) .
$$

Similarly to the stationary granular jump, one can analyse how the depth ratio of the propagating bore is influenced by the various parameters: $k_{i}, \tan \theta-\mu_{e}, \bar{K}$ and $L_{*} / h_{1}$. Such an analysis, which gives similar results to those obtained for the stationary granular jump, is not detailed here.

Eq.(13) is slighly different from Eq.(5). Figure $3 \mathrm{~b}$ shows the depth ratio $h_{2} / h_{1}$ versus $F r_{1}$ for a propagating jump [Eq.(13)] compared to a steady granular jump [Eq.(5)]. It is considered that the density variation across the jump is nil $\left(\bar{\rho}_{2} / \bar{\rho}_{1}=1\right)$ and the following values for the various parameters are used: $k_{i}=1, \bar{K}=1, L_{*} / h_{1}=10$ and $\tan \theta-\mu_{e}=0.11$. At a given value of the Froude number of the incoming flow, the propagating bore is thicker than the stationary jump. This result is not general but depends on the combination of parameters, as detailed in [57].

The equations proposed above are a slight extension of the equations which were initially proposed by Hákonardóttir [60] but did not consider the source term related to friction acting within the jump volume. Through accounting only for the source term related to the component of the gravitational acceleration along the slope, Hákonardóttir [60] distinguished two cases: the angle of repose of the granular material $\varphi$ is either smaller or greater than the slope $\theta$. The analysis by [60] leads to two schematic diagrams of the granular bore. When $\varphi \geq \theta$, the depth $h_{2}$ of the bore is constant, and the free-surface downstream of the jump is parallel to the bottom, as drawn in Fig.3a. In contrast, when $\varphi \leq \theta$, the depth $h_{2}$ is increased while the flow approaches the wall [60], and the slope of the free-surface is equal to $\varphi$. The latter situation was studied in detail by Pudasaini et al. [3] through small-scale laboratory tests. The shape of the granular bore evolving over time could be reproduced well by depth-averaged numerical simulations - including shock capturing technique - once the measured model parameters were carefully implemented, as demonstrated by Pudasaini and Kröner [4]. In their study, Pudasaini and Kröner [4] evidenced a strong influence of the internal friction angle of the granular material. 
(a)

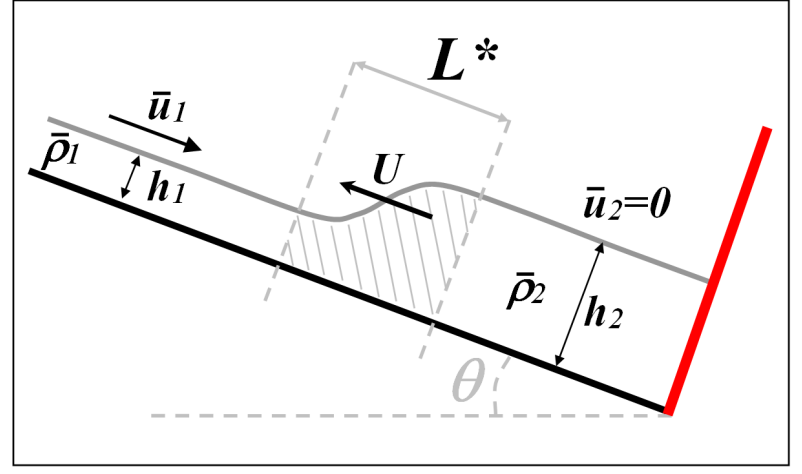

(b)

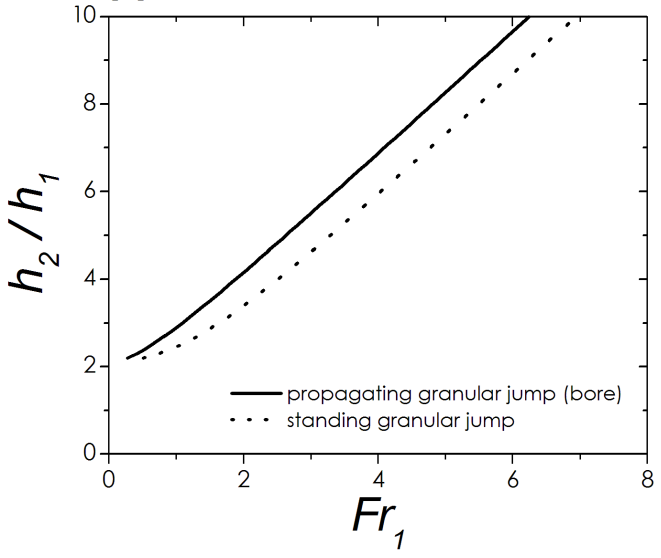

FIG. 3. a) (Color online) Sketch of a granular jump propagating upstream of a rigid wall (namely a granular bore). b) Depth ratio $h_{2} / h_{1}$ versus the Froude number $F r_{1}$ for a propagating granular jump [Eq.(13)] and for a steady granular jump [Eq.(5)]. Incompressible jumps and bores are considered $\left(\bar{\rho}_{2} / \bar{\rho}_{1}=1\right)$. Identical values of the parameters $\left(\beta_{i}=1, k_{i}=1, \bar{K}=1, L_{*} / h_{1}=10\right.$ and $\left.\tan \theta-\mu_{e}=0.11\right)$ are considered for Eqs.(13) and (5).

\section{Simplified solution for bores}

For frictionless materials $\left(\mu_{e}=0\right)$ flowing down an horizontal plane $(\theta=0)$, and under the assumption $k_{i}=1$, an estimate of the depth ratio can be obtained:

$$
F r_{1}=\frac{h_{2}}{h_{1}} \sqrt{\frac{1}{2}\left(\bar{\rho}_{2} / \bar{\rho}_{1}-\frac{1}{h_{2} / h_{1}}-\left(\frac{1}{h_{2} / h_{1}}\right)^{2}+\left(\frac{1}{h_{2} / h_{1}}\right)^{3} \frac{1}{\bar{\rho}_{2} / \bar{\rho}_{1}}\right)} .
$$

Eq.(14) with $\bar{\rho}_{2} / \bar{\rho}_{1}=1$ yields a cubic function of $h_{2} / h_{1}$ :

$$
\left(\frac{h_{2}}{h_{1}}\right)^{3}-\left(\frac{h_{2}}{h_{1}}\right)^{2}-\left(1+2 F r_{1}^{2}\right) \frac{h_{2}}{h_{1}}+1=0,
$$

which can be solved with the Cardano's method. Eq.(15) has only one physically meaningful solution, as earlier discussed by Jóhannesson et al. [61]:

$$
\frac{h_{2}}{h_{1}}=\frac{1}{3}\left(2 \cos \delta_{0} \sqrt{6 F r_{1}^{2}+4}+1\right),
$$

where $\delta_{0}$ is given by: 


$$
\delta_{0}=\frac{1}{3} \arctan \left(\frac{F r_{1} \sqrt{27\left(16+13 F r_{1}^{2}+8 F r_{1}^{4}\right)}}{9 F r_{1}^{2}-8}\right) .
$$

Note that Eqs.(16) and (17) are currently considered as important criteria in the European guidelines for the design of protection dams against avalanches [62]. However, it is worthy to stress that those equations do not take into account some important aspects of granular flows, such as possible changes in density and velocity profiles across the jump, and the effect of friction as well. The equation proposed to derive the height of a granular bore [Eq.(13)] needs to be verified on new relevant laboratory tests and/or discrete numerical simulations. Attention should be paid to low and moderate values of $F r_{1}$, and to all parameters involved in Eq.(13): $k_{i}, \bar{K}, L_{*} / h_{1}$ and $\mu_{e}$.

\section{From a granular bore (upstream of the wall) to a large airborne jet (down- stream of the wall)}

The previous section addressed the granular bore upstream of a wall while overflow downstream of the wall was prevented. One can wonder what might occur when the wall height $H$ relative to the incident flow $h_{1}$ is decreased, which causes overflow downstream of the wall. The latter situation is depicted in Fig.4a. As $H / h_{1}$ decreases, an increasing proportion of the flow can escape. Finally the bore no longer propagates upstream of the wall, and a large jet forms downstream of the obstacle, as displayed in Fig.4b. Hence, as suggested by Hákonardóttir [60], there exists a minimum height of the wall for which a bore is present upstream of the wall. A mathematical description of the bore upstream of the wall when some flow overtops the wall can be formulated indeed. A description, which accounts for the source terms (gravitational acceleration along the slope and retarding acceleration caused by friction), is proposed in the following. This is again a slight extension of the earlier analytical solution proposed by Hákonardóttir [60] for frictionless flows down a slope.

The conservations of mass and momentum fluxes across the jump in the reference frame travelling upstream with the bore at speed $U$ give (Fig.4a):

$$
\begin{array}{r}
\bar{\rho}_{1} h_{1}\left(\bar{u}_{1}+U\right)=\bar{\rho}_{2} h_{2}\left(\bar{u}_{2}+U\right), \\
\bar{\rho}_{2} h_{2}\left(\bar{u}_{2}+U\right)^{2}-\bar{\rho}_{1} h_{1}\left(\bar{u}_{1}+U\right)^{2}=\frac{1}{2} \bar{\rho}_{1} g h_{1}^{2} \cos \theta-\frac{1}{2} \bar{\rho}_{2} g h_{2}^{2} \cos \theta+W \sin \theta-F_{f},
\end{array}
$$


(a)

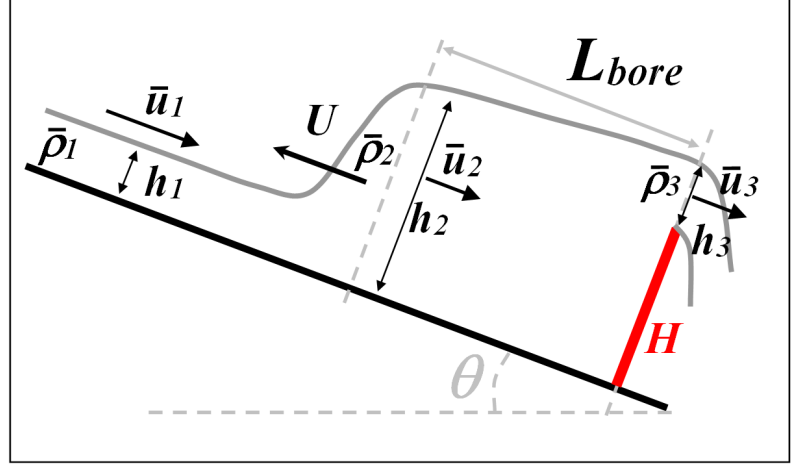

(b)

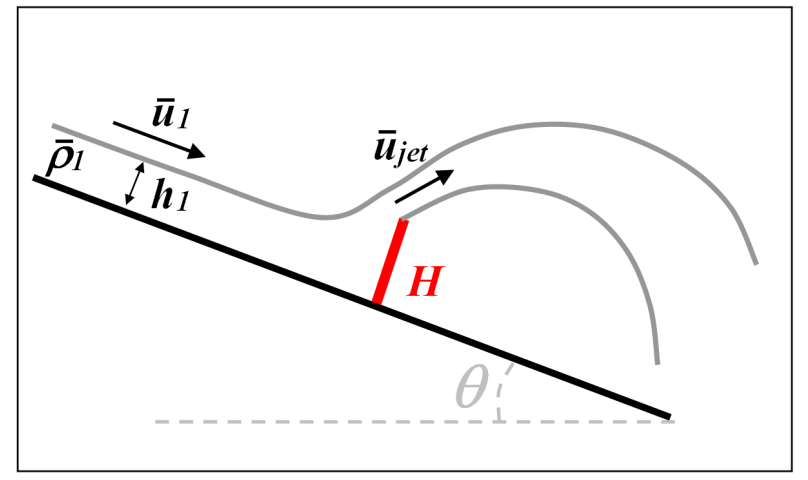

FIG. 4. (Color online) a) Sketch of a propagating granular jump (or bore) upstream of a wall with an overflow downstream of the wall; b) Large airborne jet formed downstream of the wall when the wall height is decreased and the bore no longer exists.

where notation similar to that used for the stationary granular jump equation (see Eq.(4) in section II B 1) is considered.

The mass flux conservation between the granular jump and the top of the wall gives $h_{3} \bar{u}_{3}=h_{2} \bar{u}_{2}$. Following the assumption of a critical flow at the top of the wall [60], with $F r_{3}=F r_{c}$ (it is assumed a generalized critical value of the Froude number likely to be different from 1), it yields:

$$
\bar{u}_{3}=F r_{c} \sqrt{g h_{3} \cos \theta} \text {. }
$$

As described by Hákonardóttir [60], the bore propagates upstream until the mass flux over the jump equals the mass flux over the obstacle $\left(\bar{u}_{1} h_{1}=\bar{u}_{2} h_{2}=\bar{u}_{3} h_{3}^{\text {crit }}\right)$ and the critical depth of the flow over the obstacle is then given by:

$$
h_{3}^{c r i t}=\left(\frac{\bar{u}_{1} h_{1}}{\sqrt{g \cos \theta}}\right)^{2 / 3} F_{c}^{-2 / 3},
$$

by considering the critical value $F r_{c}$ of the Froude number, which can be different from 1 for frictional granular flows down inclines. The lower the obstacle height is, the more mass is transported over the obstacle, and when:

$$
\frac{H}{h_{1}} \leqslant \frac{h_{2}}{h_{1}}-\frac{h_{3}^{c r i t}}{h_{1}},
$$


all the approaching mass flux is transported directly over the obstacle and the bore does not need to propagate upstream. In this context, ones moves from a granular bore to a standing granular jump $[U=0$ in Eqs.(18) and (19)]. The critical wall height below which the bore does not exist is then given by the following relation:

$$
\frac{H_{c}^{j e t}}{h_{1}}=\frac{h_{2}}{h_{1}}-\left(\frac{F r_{1}}{F r_{c}}\right)^{2 / 3},
$$

where the ratio $h_{2} / h_{1}$ is given by Eq.(5) (full equation) or Eq.(7) (approximate solution for incompressible granular fluids). Eq.(23) includes the effect of the slope and the effective friction, and will be used to interpret some of the existing experimental data described in section III B.

The propagation of granular bores at high slope over a smooth bottom (meaning that the typical size of roughness is much smaller than the grain size) has been studied by laboratory tests [3] and successfully captured by depth-averaged numerical simulations [4], provided that the constant friction parameters, measured independently, were carefully implemented in the numerical model. It would be of interest to design new tests on the propagation of bores on lower slopes, and/or over rough bottoms. The latter conditions would reduce the Froude number of the flows and produce more elongated and diffuse bores. It is likely that depth-averaged models implemented with constant friction parameters would not describe those diffuse bores as well as they can do for bores in very fast granular flows.

\section{EXPERIMENTS ON GRANULAR PATTERNS NEXT TO WALLS REVIS- ITED}

\section{A. The granular jump}

The stationary jumps and the propagating jumps (or bores) generated by an obstruction normal to the incident flow have been investigated by several authors, from the pioneering works of Morrison and Richmond [54], Savage [55], Brennen et al. [63], Campbell et al. [64] to more recent studies $[3-5,10,20,56,60,65]$. The following sub-sections revisits some of the existing data on granular jumps, in line with the analytic solutions proposed in section II. 


\section{The stationary granular jump}

Brennen et al. [63] distinguished two types of stationary granular jumps and identified three types of flows between the jump and the wall (flat plate weir in their tests). At low $F r_{1}$, the flow in the jump consisted of a smooth expansion whereas, at high $F r_{1}$, the jump contained a recirculating eddy necessarily accompanied by a stagnation line. The smooth expansion, namely the very diffuse jump, characterized by a low value of the ratio $h_{2} / h_{1}$ would correspond to the so-called granular dead zone process described by Faug et al. [66], as it will be discussed in section III C. At the lowest slopes, the flow between the jump and the weir was shearing over its entire depth. At highest slopes, the jump was followed immediately by the formation of a base wedge. An intermediate situation corresponded to a more complex jump structure, as detailed by Brennen et al. [63]. However, Brennen et al. [63] found that the comparison between theory and their experimental data was not affected by the type of jump (smooth expansion or jump with recirculating eddy) or of downstream flow. Their experimental data were compatible with data reported earlier by Savage [55].

Figure 5 shows the depth ratio $h_{2} / h_{1}$ measured by Savage [55] and Brennen et al. [63] as a function of $F r_{1}$. Faug et al. [56] have recently designed a laboratory chute equipped with a discharge gate at the exist of the chute, able to produce standing jumps over a wide range of slope angle and mass discharge. They identified compressible jumps for which the density variation across the jump cannot be neglected. Above a critical mass discharge for which the incoming flow are not too dilute, the jumps were incompressible. Their data on incompressible jumps is also included in Fig.5.

The collection of data available in literature $[55,56,63]$ show that all data merge into one single group. The prediction from the hydraulic jump equation, namely $h_{2} / h_{1}=$ $\left(\sqrt{1+8 F r_{1}^{2}}-1\right) / 2$, which is strictly valid for frictionless flows down a horizontal plane, is also shown by the line drawn in Fig.5. The line is systematically below the laboratory data. This result clearly highlights the need of an equation suitable for granular media.

As a first step, and under the assumption of incompressible jumps $\left(\bar{\rho}_{2} / \bar{\rho}_{1}=1\right)$, Eq.(7) is fitted on all laboratory data reported in Fig.14. The following parametrization is considered:

$$
\frac{h_{2}}{h_{1}}=\frac{1}{2}\left(\sqrt{1+8 \chi F r_{1}^{2}}-1\right) \text {, }
$$




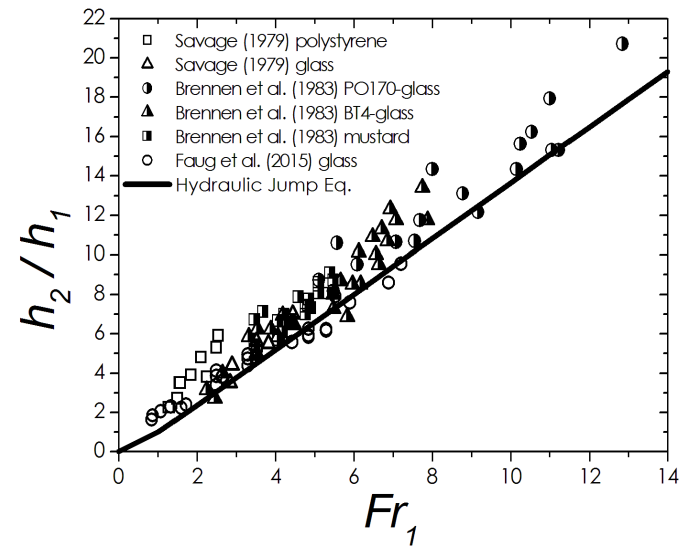

FIG. 5. $h_{2} / h_{1}$ versus $F r_{1}$ measured experimentally by Savage [55], Faug et al. [56], Brennen et al. [63]. The line shows the prediction from the traditional hydraulic equation $h_{2} / h_{1}=\left(\sqrt{1+8 F r_{1}^{2}}-\right.$ $1) / 2$.

where $\chi=\beta /\left[k-\frac{\bar{K} L_{*}\left(\tan \theta-\mu_{e}\right)}{\left(h_{2}-h_{1}\right)}\right]$ is used as a tuning parameter. Figure 6 depicts the exact values for $\chi$ as a function of the Froude number for each couple $\left(F r_{1}, h_{2} / h_{1}\right)$ measured by Savage [55], Faug et al. [56], Brennen et al. [63]. Though the data remain scattered, one can note the following trend: $\chi$ is close to 1 at high $F r_{1}$ while it starts diverging when $F r_{1}$ is decreased. This is the proof of a transition-which occurs around $F r_{1} \sim 3-4$, as clearly demonstrated by the recent experiments by Faug et al. [56]. By decreasing $F r_{1}$, there is a transition from granular jumps (in rapid flows) relatively well predicted by the traditional hydraulic equation toward granular jumps (in slower flows) whose height is much higher than the height predicted by the traditional hydraulic equation. Fig.6 shows that this finding could be extracted from the existing data provided in earlier studies by Savage [55] and Brennen et al. [63].

In contrast to previous studies by Savage [55] and Brennen et al. [63], Faug et al. [56] paid a detailed attention to the shape of granular jumps and found that the jumps were steep at high $F r_{1}$, while they became much more diffuse at lower $F r_{1}$, as depicted in the pictures provided in Fig.7.

Fig.8a and b summarize the key experimental results by Faug et al. [56] through depicting how the shape coefficient $\bar{K}$ and the ratio $L_{*} /\left(h_{2}-h_{1}\right)$ evolve with $F r_{1}$ (see details in [56] on how the jump length $L_{*}$ was defined experimentally). $\bar{K}$ increases roughly linearly with $F r_{1}$, 


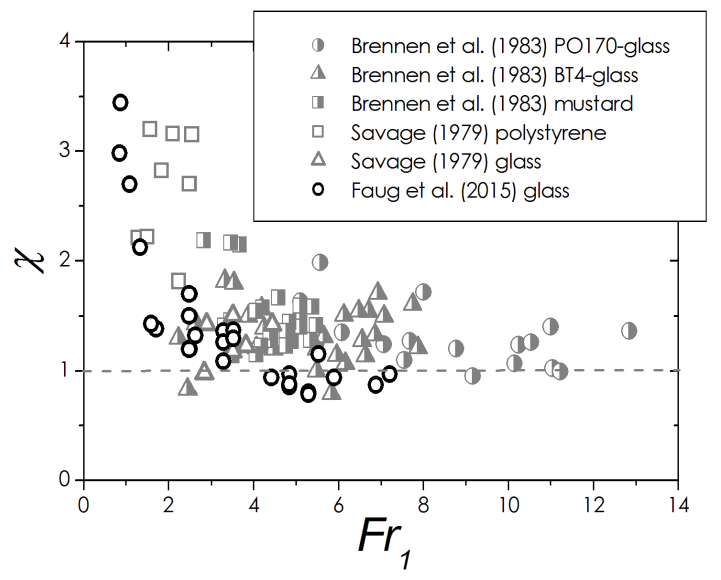

FIG. 6. Exact value of $\chi$ as a function of the Froude number from the cross-comparison between Eq.(24) and the laboratory data of Savage [55], Faug et al. [56], Brennen et al. [63]. The line $\chi=1$ would correspond to the prediction of the traditional hydraulic equation.
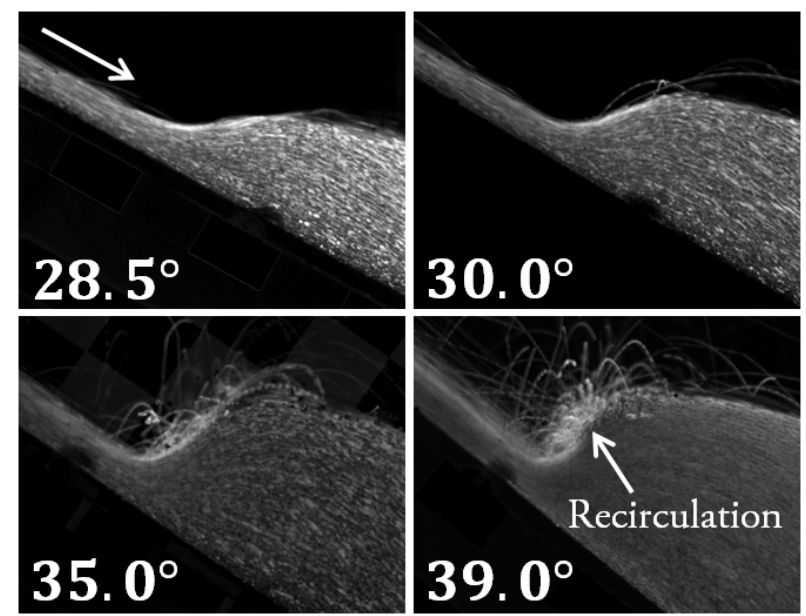

FIG. 7. Transition observed by Faug et al. [56] between steep jumps at high $F r_{1}$ (high slope) and diffuse jumps at lower $F r_{1}$ (lower slope). Some recirculation was observed at the highest slopes.

while $L_{*} /\left(h_{2}-h_{1}\right)$ depicts a sharp increase when the Froude number decreases. By fitting Eq.(7) (with $k=1$ and $\beta=1$ ) to the experimental data, the friction $\mu_{e}$ could be derived (see detail in [56]) and compared to $\tan \theta$, as shown in Fig.8c. The difference $\tan \theta-\mu_{e}$ remains tiny and weakly influenced by $F r_{1}$ (a small increase with $F r_{1}$ may be detectable but remains within the uncertainty of measurements), as drawn in Fig.8d. From the curves drawn in Fig.8, it can be concluded that the dramatic increase of $L_{*} /\left(h_{2}-h_{1}\right)$ when $F r_{1}$ is 
below $3-4$ prevails. This produces an increase of the product $\bar{K} \frac{L_{*}}{\left(h_{2}-h_{1}\right.}\left(\tan \theta-\mu_{e}\right)$, which makes $\chi$ higher than 1 in Eq.(24). The height of the diffuse jumps is then higher than the height predicted by the traditional hydraulic equation. This result is interpreted as a direct consequence of the forces caused by the gravity acceleration balanced by friction, and acting within the jump volume. Not considering those forces would lead to an underestimate of the height of the diffuse granular jumps observed at low $F r_{1}$, typically smaller than $3-4$. The traditional hydraulic equation is only suitable for (incompressible) granular jumps at high $F r_{1}$. Predicting the height of granular jumps at low $F r_{1}$ is yet a problem to be fully solved for two reasons: (i) Eq.(7) remains implicit $\left(h_{2} / h_{1}\right.$ being a function of $\left.h_{2}-h_{1}\right)$ and (ii) it does not exist any theory to predict the length of the jump $L_{*}$.

In want of a better knowledge of the geometry of jumps, one can propose an empirical approach by fitting the following relations on the measured values of $\bar{K}$ and $L_{*} /\left(h_{2}-h_{1}\right)$, which are assumed to depend primarily on $F r_{1}$ (as usually stated in hydraulics [53]):

$$
\begin{gathered}
\bar{K}=1+a\left(F r_{1}-F r_{c}\right), \\
\frac{L_{*}}{h_{2}-h_{1}}=\frac{b}{\left(F r_{1}-F r_{c}\right)^{c}}
\end{gathered}
$$

where $a, b$, and $c$ are constant parameters. The relation for $L_{*} /\left(h_{2}-h_{1}\right)$ is a power law able to capture the increase of $L_{*} /\left(h_{2}-h_{1}\right)$ when $F r_{1}$ approaches the critical value $F r_{c}$. Note that $F r \mapsto F r_{c}$, yielding $h_{2} \mapsto h_{1}$, is a singular point. Describing what happens at tiny values of $h_{2}-h_{1}$ is challenging because the processes are strongly driven by the complex interplay between hydrodynamic properties and the frictional nature of the granular fluid. The relation for $\bar{K}$ is an affine function which satisfies the following property: $\bar{K} \mapsto 1$ when $F r \mapsto F r_{c}$ (very diffuse jumps). The fitted lines for $\bar{K}$ and $L_{*} /\left(h_{2}-h_{1}\right)$ are reported in Fig.8a and b, respectively, and the values of parameters $a, b$ and $c$ are indicated as well. $F r_{c}=0.6$ provided the best fits. Using $F r_{c}=1$ does little to change the fitted curves but it is not consistent with the laboratory results by Faug et al. [56] who could observe very diffuse jumps at values of $F r_{1}$ lower than 1 . In want of any further knowledge at the present time, the empirical relations for $\bar{K}$ and $L_{*} /\left(h_{2}-h_{1}\right)$ will be used in the following to estimate the bore height and the critical wall height below which jumps no longer exist. 

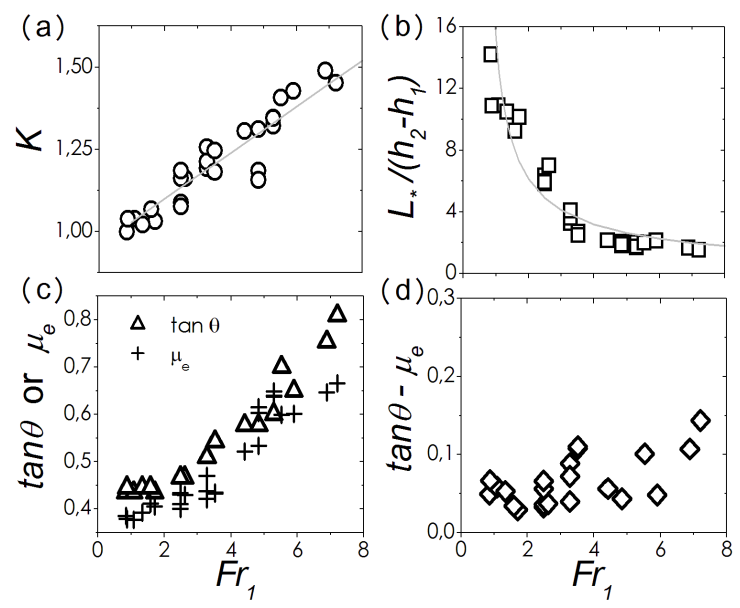

FIG. 8. (a) $\bar{K}$ versus $F r_{1}$; the continuous line shows the fit with $1+a\left(F r-F r_{c}\right)$, where $a=0.07$ and $F r_{c}=0.6$. (b) $L_{*} /\left(h_{2}-h_{1}\right)$ versus $F r_{1}$; the continuous line shows the fit with $\frac{b}{\left(F r-F r_{c}\right)^{c}}$, where $b=8, c=0.75$ and $F r_{c}=0.6$. (c) $\tan \theta$ and $\mu_{e}$ versus $F r_{1}$. (d) $\tan \theta-\mu_{e}$ versus $F r_{1}$ (summary of laboratory data by Faug et al. [56]).

\section{Bores and critical wall height below which jumps no longer exist}

In particular studies conducted in geophysics to understand the interaction between avalanches of granular materials and walls, the key role played by the occurrence - or the non-occurrence - of granular bores has been pointed out. Hákonardóttir [60] and Faug et al. [10] investigated the impact of granular avalanches with walls that mimick avalanche barriers, and observed different granular patterns depending on the wall height relative to the incoming flow depth.

Faug et al. [10] designed laboratory tests on a granular avalanche $\left(F r_{1} \sim 3.75\right)$, which overflowed dams of various heights from $H / h_{1}=0.7$ to $H / h_{1}=7.4$. Those tests were initially performed to study avalanche run-out shortening caused by dams, which is crucial for avalanche protection in geophysics [6, 8-10, 67]. In their experiments, Faug et al. [10] observed a distinct transition, around $H / h_{1}=2-3$, from a gentle overflow of the granular material to a bore regime (see figure 4 in [10]). A sharp jump propagated far upstream of the wall in the latter situation. In the former situation, a quasi-steady dead zone formed against the wall and other incoming grains were able to pass smoothly on the dead zone to overflow the wall. This situation is discussed in more detail in section III C. Eq.(23) that 
predicts the critical wall height $H_{c}^{j e t}$ below which bores no longer exist can be checked against the experimental observations by [10]. Each couple $\left(F r_{1}, H / h_{1}\right)$ tested by [10] is reported in Fig.10a, by distinguishing between the experiments with a bore propagating upstream (crosses) and the ones without any bore (circles). The prediction from Eq.(23) is reported as well, where $h_{2} / h_{1}$ is calculated with the approximate solution given by Eq.(7). The following values were considered for the parameters needed: $k=1, \beta=1$, and $L_{*} /\left(h_{2}-h_{1}\right)$ and $\bar{K}$ were derived from the empirical relations proposed in Sec III A 1. Two values of the difference $\tan \theta-\mu_{e}$ were tested: 0.05 and 0.10 , which are typical values recently measured by [56] (see Fig.8d). Eq.(23) is found to be quite efficient at demarcating the experiments with the sharp jump (crosses in Fig.10a) from the experiments with the more diffuse overflow (circles in Fig.10a).

Hákonardóttir [60] designed laboratory tests on much more rapid granular avalanche $\left(F r_{1} \sim 12\right)$ impacting dam-like obstacles of four different heights: (i) $H / h_{1}=H_{\infty} / h_{1}$ ( $H=H_{\infty}$ prevented overflow), (ii) $H / h_{1}=18$, (iii) $H / h_{1}=15$, and (iv) $H / h_{1}=6.7$. As shown in Fig.9b, a granular jump propagating upstream was observed for test (i) for which the incoming flow was entirely blocked upstream of the dam $\left(H=H_{\infty}\right)$. For tests (ii) and (iii), the granular bore was still present, while some material was able to overflow the smaller dam. In contrast, a long airborne granular jet formed downstream of the dam in test (iv), when $H / h_{1}$ was reduced to 6.7 . Eq.(23) that predicts the critical wall height $H_{c}^{\text {jet }}$ below which bores no longer exist can be checked against the experimental observations by Hákonardóttir [60]. Fig.10a shows each point $\left(F r_{1}, H / h\right)$ corresponding to tests (ii)(iv) conducted by Hákonardóttir [60]. The prediction from Eq.(23) is reported as well. Again, the following values were considered for the parameters needed: $k=1, \beta=1$ and $L_{*} /\left(h_{2}-h_{1}\right)$ and $\bar{K}$ were derived from the empirical relations proposed in Sec III A 1. Two values of $\tan \theta-\mu_{e}$ were tested: 0.05 and 0.10. Again, Eq.(23) allows to demarcate roughly the experiments with bores [tests (ii) and (iii) represented by cross symbols] from the experiment without a bore and a large jet formed downstream of the wall [test (iv) shown in a circle symbol].

It is worthy to note that the predictions from traditional hydraulics $\left(F r_{c}=1\right.$ and $\tan \theta-$ $\mu_{e}=0$ ), reported in dashed lines in Fig.10, are very close to predictions from the approximate solution stemming from Eq.(7) implemented with the empirical laws for $\bar{K}$ and $L_{*} /\left(h_{2}-h_{1}\right)$. They are thus efficient at capturing the transitions observed by [10, 60] for experiments at 

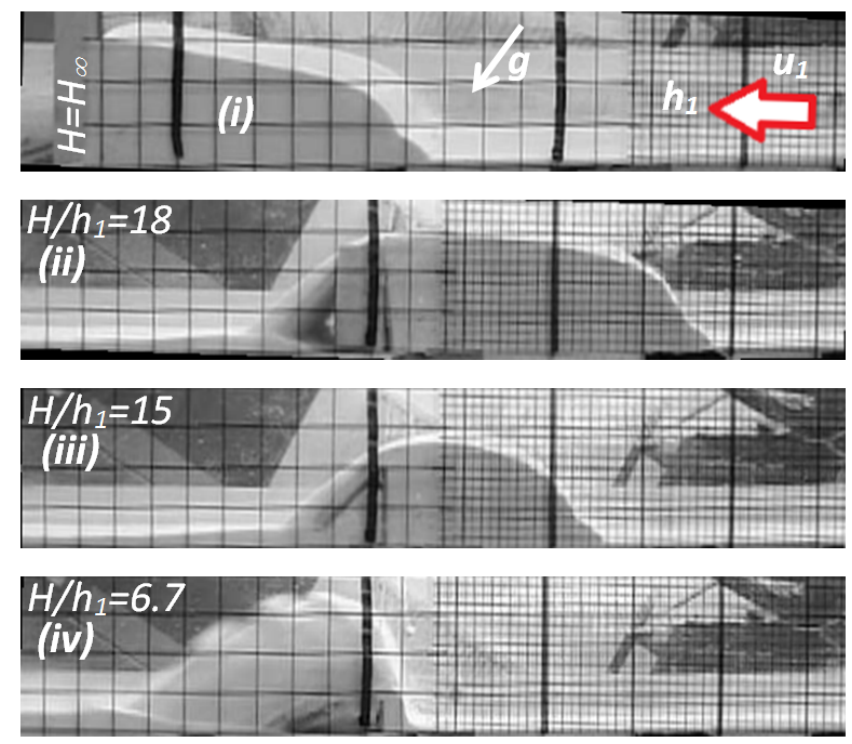

FIG. 9. (Color online) Hákonardóttir [60] studied granular flows at high Froude number $\left(F r_{1} \sim 12\right)$ impacting a rigid wall. At large wall height preventing overflow, a granular jump propagate upstream [test(i)]. While decreasing the wall height, a clear transition from a granular bore propagating upstream of the wall with some overflow downstream of the wall [tests (ii) and (iii)], to a large airborne jet formed downstream of the dam [test (iv)] was observed. The photographs by K.M. Hákonardóttir were adapted from Hákonardóttir [60].

high Froude numbers. However, it is likely that hydraulic theory would fail for lower values of the Froude number for which the term $\bar{K} \frac{L_{*}}{h_{2}-h_{1}}$ starts playing a role in Eq.(7).

\section{B. The granular airborne jet}

Granular airborne jets are observed in very rapid granular flows $\left(F r_{1} \gg 1\right)$ interacting with a relatively small wall $\left(H / h_{1} \ll 1\right)$, as shown in Fig. 11a. The length of the influence zone upstream of the obstacle is small and reduced to a small wedge of stagnant grains (the size of the wedge is typically close to the wall height). In geophysics, the length of granular airborne jet is for instance relevant for the design of the minimum distance between two rows of dissipative structures against snow avalanches [26, 68]. Predicting accurately the jet length avoids designing dissipating structures that are ineffective because they would be overtopped by the jet. 

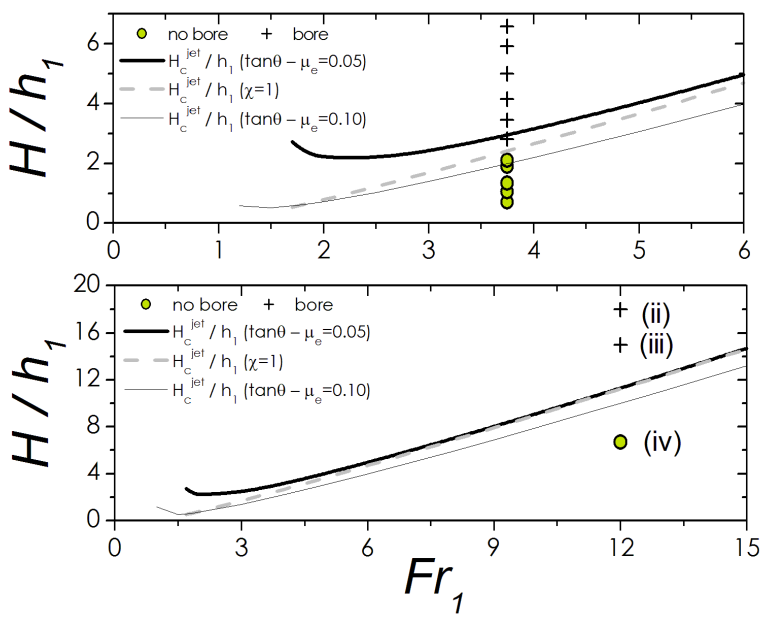

FIG. 10. Points $\left(F r_{1}, H / h_{1}\right)$ tested by Faug et al. [10] (top graph) and Hákonardóttir [60] (bottom graph). Cross symbols $(+)$ correspond to tests for which a bore propagated upstream and circle symbols (o) represent tests for which the bore was not present. The continuous lines show predictions from Eq.(23) implemented with Eq.(7) for various values of $\tan \theta-\mu_{e}$ (see text). The dashed lines show the prediction from traditional hydraulics for horizontal flows.

The trajectory of these airborne jets was studied in detail by Hákonardóttir et al. [13] (note that airborne snow jets have been also studied [26]). The experiments showed that the rapid flow detached from the top of the obstacle as a coherent granular jet, the motion of which being well described by theory for a two-dimensional irrotational flow of an inviscid fluid over a dam [69]. As suggested by Hákonardóttir [60], the theory from Yih [69] gives a relation between the launch angle $\beta_{j e t}$, the angle of the uptream face of the wall $\alpha$ and the obstacle height relative to the incoming flow $H / h_{1}$. Notation used is shown in Fig.11a, and further details for the theory of Yih can be found in [60]. Hákonardóttir et al. [13] showed that on colliding with a barrier, a shallow granular flow of high Froude number becomes airborne and follows a coherent ballistic trajectory, with negligible effect of resistive forces within the deflection region. Thus, for a given flow speed and depth of the avalanche relative to that of the obstacle, the length of the airborne jet can be estimated by combining the prediction of the angle of deflection $\beta_{\text {jet }}$ with the parabolic trajectory of the jet, on the assumption that air resistance plays only a negligible role for these trajectories (note that similar conclusion was obtained for rapid snow-chute flows [26]). 

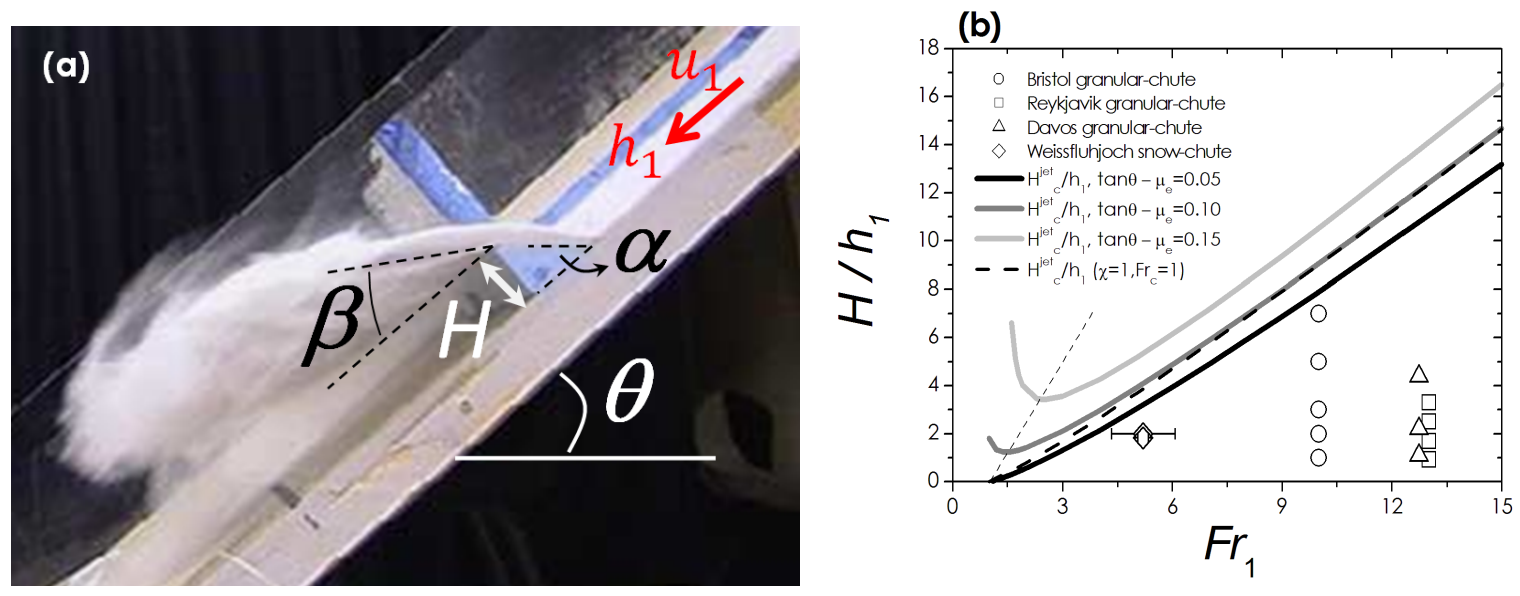

FIG. 11. a) (Color online) Photograph of the granular jet as it detaches from the top of the obstacle (photograph by K.M. Hákonardóttir); b) Values of the couple $\left(F r_{1}, H / h_{1}\right)$ tested in the laboratory experiments on airborne jets with granular materials [13] and snow [26]. The predictions from Eq.(23) for the critical height $H_{c}^{j e t}$, below which the granular bore no longer exists, are drawn for $k=1$ and $\beta=1 . L_{*} /\left(h_{2}-h_{1}\right)$ and $\bar{K}$ are derived from the empirical functions of $F r_{1}$. Three different values are considered for $\tan \theta-\mu_{e}: 0.05,0.10$ and 0.15 . The dashed line shows the prediction from traditional hydraulics.

Figure 11b displays the experimental values of the couple $\left(F r_{1}, H / h_{1}\right)$ tested by Hákonardóttir et al. [13] (note that tests from snow-chute flows are also included [26]). Those values correspond to tests for which large airborne granular jets were observed downstream of the wall, without any granular jump formed upstream of the wall, thus offering a test for Eq.(23) described in Sec. II D. Eq.(23) gives the critical wall height, $H_{c}^{j e t}$, which demarcates the granular bores propagating upstream of the wall from the large airborne jets formed downstream without any granular jump formed upstream. The prediction of that equation should be above all the points corresponding to couples $\left(F r_{1}, H / h_{1}\right)$ tested by Hákonardóttir et al. [13, 26]. The values of each parameter needed for Eq.(23) implemented with Eq.(7) are given in caption of Fig.11. It is verified that all the points corresponding to couples $\left(F r_{1}, H / h_{1}\right)$ tested by Hákonardóttir et al. [13], and by Hákonardóttir et al. [26] for snow-chute flows, are below the prediction of $H_{c}^{j e t}$ shown by the group of lines drawn in Fig.11b. 

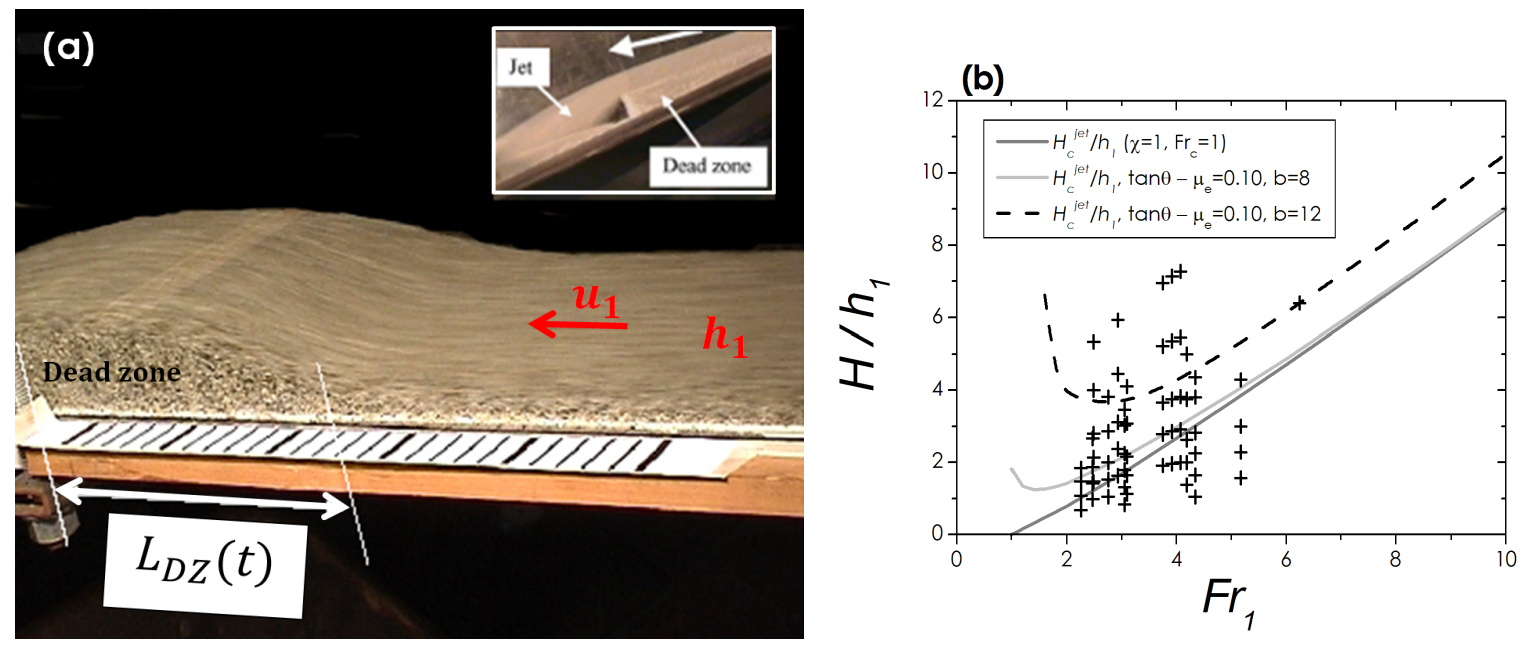

FIG. 12. a) (Color online) Photograph of the granular dead zone created upstream of a wall: creation of the dead zone (Inset: stationary dead zone [66]); b) Values of couples $\left(F r_{1}, H / h_{1}\right)$ which correspond to the experiments on dead zones by Faug et al. [66]. The predictions from Eq.(23) for the critical height $H_{c}^{\text {jet }}$, below which the granular bore no longer exists, are drawn for $k=1, \beta=1$ and $\tan \theta-\mu_{e}=0.15 . L_{*} /\left(h_{2}-h_{1}\right)$ and $\bar{K}$ are derived from the empirical functions of $F r_{1}$. Two values of the $b$-constant are considered: $b=8$ and $b=12$. The prediction from traditional hydraulics is reported too.

\section{The granular dead zone regime}

Relatively slow flows $\left(F r_{1} \sim 1\right)$ impacting relatively small walls $\left(H / h_{1} \sim 1\right)$ produce the formation of a quasi-static stagnant zone upstream of the wall, which can reach a stationary length, while the incoming grains start to flow gently over the static pile and form a stationary jet whose energy is much smaller than the airborne jet discussed in section III B. This granular pattern is illustrated in Fig.12a (see also Fig.9b).

The co-existence between this stagnant zone and the flowing grains above the dead zone has been observed and studied in detail by Faug et al. [66]. This so-called dead zone process $[28,66,70]$ differs from the granular jump because it is not accompanied by a sharp discontinuity in depth (strong jump). It consists more of a dense granular stream flowing down a pile made of quasi-static grains. The thickness and the depth-averaged velocity, and the density as well, of the flowing zone vary smoothly. The values of couples $\left(F r_{1}, H / h_{1}\right)$ tested by Faug et al. [66] are drawn in Fig.12a. The reasons why such a dead 
zone (without any sharp jump formed) was observed even for relatively high $F r_{1}$ and $H / h_{1}$ in the experiments by Faug et al. [66] still remains unclear. However, it is worthy to note that increasing $b$, which corresponds to an increase of the length of the jump, produces an increase of $H_{c}^{j e t}$. Increasing $H_{c}^{j e t}$ then leads to an increase of the area for which dead zones (in absence of a bore) can be observed. The predictions from Eq.(23) for the critical height $H_{c}^{j e t}$, below which the granular bore no longer exists, are drawn in Fig. $12 \mathrm{~b}$ for $k=1, \beta=1$ and $\tan \theta-\mu_{e}=0.15 . L_{*} /\left(h_{2}-h_{1}\right)$ and $\bar{K}$ are derived from the empirical functions of $F r_{1}$. Two values of the $b$-constant are considered: $b=8$ and $b=12$.

Fig.13 displays the measured length of the dead zone [66] scaled by the thickness of the incident flow, $L_{d z} / h_{1}$, as a function of the Froude number of the obstacle, $F r_{H}=\bar{u} / \sqrt{g H \cos \theta}$. $F r_{H}$ is defined by $F r_{1}^{2}=F r_{H}^{2}\left(H / h_{1}\right)$. Fig.13 shows that $L_{d z} / h_{1}$ is strongly correlated to $F r_{H}$, which demonstrates the importance of the dimensionless number $F r_{H}$. For $F r_{H} \sim 1$, the dead zone length starts diverging. For the largest values of $F r_{H}, L_{d z} / h_{1}$ reaches a constant value around 10. Most of the studies about flow-wall interactions investigated rapid granular flows down steep slopes and/or over smooth bottoms (typical size of roughness much smaller than the grain size). That rapid dynamics remains of course important but attention should be paid to the slower dynamics too. New tests should be designed to investigate slow granular flows on lower slopes and/or over a rough bottom. The dead zone process stems from the ability of granular materials to form stagnant zones (solid-like behaviour) which can co-exist with inertial zones (fluid-like behaviour). It is worthy to note that recent developments have been conducted to predict the force experienced by the wall when a dead zone is present, as discussed in [71] and references therein.

\section{DISCUSSION AND OUTLOOK}

The analytic solutions presented in the present study reveal the crucial importance of the following depth-averaged dimensionless numbers needed to describe how the geometry of the granular flow is influenced by the presence of a wall: (i) the Froude number $F r_{1}=$ $\bar{u}_{1} / \sqrt{g h_{1} \cos \theta}$ of the incident flow and (ii) the obstacle height relative to the incoming flow depth $H / h_{1}$. The Froude number of the obstacle $F r_{H}=\bar{u}_{1} / \sqrt{g H \cos \theta}$ is also a dimensionless

number of interest, which depends on the two aforementioned numbers. The difference between $\tan \theta$ and the effective basal friction $\mu_{e}$, and the earth pressure coefficient $k$ are 


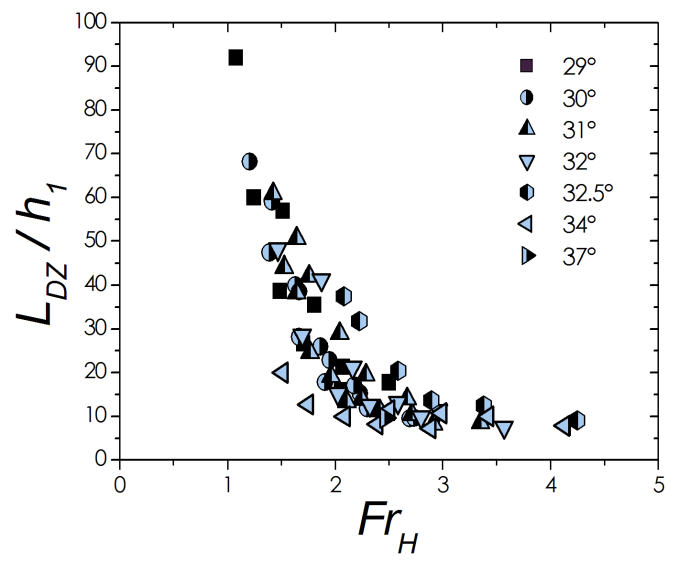

FIG. 13. Dead zone length scaled by the thickness of the incident flow, $L_{d z} / h_{1}$, versus the Froude number of the obstacle, $F r_{H}$, as measured by Faug et al. [66].

additional dimensionless parameters to take into account the granular nature of the fluid at stake. Under specific conditions (steep slope and smooth bottom, which lead to jumps short in length), $\mu_{e}=\tan \delta$ may be a good approximation. More complex effective friction laws, for which $\mu_{e}$ depends on depth-averaged flow variables $h_{1}$ and $\bar{u}_{1}$, need the introduction of other dimensionless numbers [46-51].

Let's define $L_{+}$and $L_{-}$the length of the zones of influence upstream and downstream of the wall, respectively. $d$ is the grain diameter. Both $L_{+} / d$ and $L_{-} / d$ are functionsrespectively called $f$ and $g$-of $F r_{1}$ and $H / h_{1}$, and $L_{+} / d$ may be additionnally influenced by $\mu_{e}, \varphi$ and $k$ :

$$
\begin{array}{r}
\frac{L_{+}}{d}=f\left(F r_{1}, \frac{H}{h_{1}}, \mu_{e}, \varphi, k\right), \\
\frac{L_{-}}{d}=g\left(F r_{1}, \frac{H}{h_{1}}\right)
\end{array}
$$

Including any influence of $\mu_{e}, \varphi$ and $k$ on $L_{-} / d$ is considered as negligible-or even irrelevant, because granular flows downstream of the wall essentially consist of detached jets formed of free falling particles in a relatively dilute regime. The three important steady regimes are defined below depending on the magnitude of the scaled lengths $L_{+} / d$ and $L_{-} / d$, and the value of $F r_{H}$ as well. This allows establishing a complete phase-diagram of the granular flow-wall interaction. 


\section{A. The granular airborne jet regime: $L_{-} / d \gg L_{+} / d\left(F r_{H} \ll 1\right)$}

At low $H / h_{1}$ and high $F r_{1}$, the incoming flow is able to flow easily over the wall forming a large granular airborne jet downstream of the wall, as depicted in Fig.4b. A steady state can be achieved. $L_{-} / d$ is high and approximately determined by the length of the stationary airborne jet formed downstream of the wall, while $L_{+} / d$ is small (typically close to the wall height from laboratory tests: $L_{+} \sim H$ ) and roughly reduced to the length of the stationary wedge formed upstream of the wall. The airborne jet regime, mainly controlled by the inertia of the incoming flow, is defined by $L_{-} / d \gg L_{+} / d$ and $F r_{H} \ll 1$.

\section{B. The granular dead zone regime: $L_{-} / d \sim L_{+} / d\left(F r_{H} \sim 1\right)$}

At intermediate values of $H / h_{1}$ and $F r_{1}$, all typical energies involved in the flow-wall interaction (both potential and kinetic energies of the incident flow, and the potential energy based on the wall height $H$ as well) are of the same magnitude. A steady-state pattern can be formed: it consists of a quasi-static stagnant zone which co-exists with an inertial flowing zone above able to flow over the obstacle, as displayed in Fig.12a. $L_{+} / d$ is determined by the length of the dead zone formed upstream of the wall, while $L_{-} / d$ corresponds to a jet formed upstream of the wall. The jet formed is shorter, denser and much less energetic than the granular airborne jet discussed above. The dead zone regime is defined by $L_{+} / d \sim L_{-} / d$ and $\mathrm{Fr}_{H} \sim 1$.

\section{The granular jump regime: $L_{-} / d \ll L_{+} / d\left(F r_{H}\right.$ undetermined $)$}

At high $F r_{1}$ and $H / h_{1}$, the incoming flow has a strong kinetic energy but the potential energy associated with the obstacle height $(\sim g H)$ is simultaneously high, which makes $F r_{H}$ undetermined. A thick layer of grains is able to propagate upstream of the wall (subcritical flow), and encounters the grains from the incoming rapid flow (supercritical flow). A granular bore, at the sharp transition between the sub-critical flow (propagating upstream) and the supercritical (incoming) flow, is formed, as depicted in Fig.4a. The distance at which the bore is able to propagate upstream defines $L_{+} / d$. Under certain conditions $\left(H<H_{c}^{j e t}\right)$,

a stationary granular jump can be formed leading to a steady value of $L_{+} / d$ while a part of the incoming flow is able to overtop the wall, forming a jet of very low energy $\left(L_{-} / d\right.$ much 
smaller than $\left.L_{+} / d\right)$.

\section{Phase-diagram for granular flow-wall interaction}

In line of the above qualitative considerations and the analytic solutions discussed in the present study, a phase-diagram for granular flow-wall interaction can be proposed, as drawn in Fig. 14.

Eq.(7) gives the scaled height $h_{2} / h_{1}$ of the steady granular jumps. Eq.(23) makes it possible to predict the critical wall height $H_{c}^{j e t}$, which defines the frontier between large airborne jets $\left(F r_{1}>F r_{c}\right.$ and $\left.H<H_{c}^{j e t}\right)$ and steady granular jumps $\left(F r_{1}>F r_{c}\right.$ and $\left.H>H_{c}^{j e t}\right)$. There exists a line above which steady-state conditions cannot be achieved and a granular bore will propagate upstream of the wall provided that the Froude number is larger than $F r_{c}$. This line corresponds to the jump height defined by Eq.(7). Once $H / h_{1}$ is larger than $h_{2} / h_{1}$, a bore propagates upstream and its height is given by Eq.(13). Below $F r_{c}$, the granular bore does not form. Still below $F r_{c}$ but at sufficiently low values of $H / h_{1}$ $\left(H<H_{c}^{j e t}\right.$ ), a steady-state can be reached through the formation of a granular dead zone co-existing with the flowing grains above able to overtop the wall.

The following values of parameters are used for the predictions given in Fig.14 [Eq.(7) for $h_{2} / h_{1}$ and Eq.(23) for $\left.H_{c}^{j e t}\right]: k=1, \beta=1$, and $\tan \theta-\mu_{e}=0.10 . \bar{K}$ and $L_{*} /\left(h_{2}-h_{1}\right)$ were derived from the empirical functions of $F r_{1}$. Two values of $b$ in Eq.(26) were considered for $H_{c}^{j e t}$ and $h_{2} / h_{1}: b=8$ (dashed lines) and $b=12$ (dotted lines). The prediction from traditional hydraulics for horizontal flows is also reported for $H_{c}^{j e t}$ (continuous line).

The phase-diagram proposed in Fig.14 was established with the help of depth-averaged analytic solutions for free-surface gravity-driven granular flows interacting rigid walls down inclines. An effort was made to systematically include the effects of the gravitational acceleration along the slope, and of the retarding acceleration caused by friction as well. This phase-diagram should be very useful for the design of future research studies on the problem of obstacles impacted by granular flows.

Empirical closure relations were used to estimate the geometry of the granular jump, i.e. the relations for $\bar{K}$ and $L_{*} /\left(h_{2}-h_{1}\right)$, and the difference $\tan \theta-\mu_{e}$ was considered as a constant in the range $0.05-0.15$, in accordance to recent laboratory results by [56]. At high Froude numbers, the semi-empirical solutions obtained provide results which are close to the 


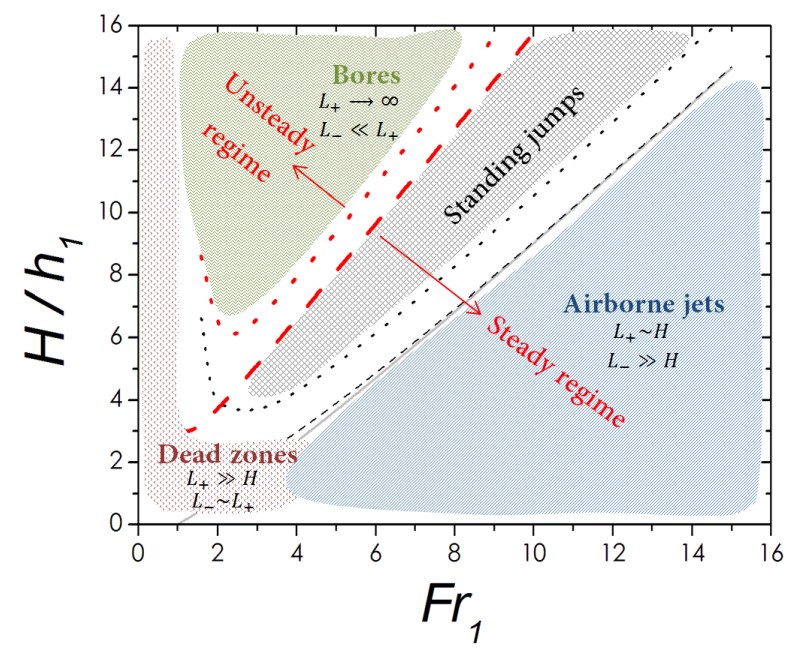

FIG. 14. (Color online) Phase-diagram for granular flow-wall interaction. The zones defining the conditions under which each of the granular patterns discussed in the present paper are drawn in the plane $\left(F r_{1}, H / h_{1}\right)$. Predictions from Eq.(7) for the height of steady jumps (thick lines) and from Eq.(23) for the critical height below which the granular bore no longer exists (thin lines) are reported $\left(k=1, \beta=1\right.$ and $\left.\tan \theta-\mu_{e}=0.10\right) . \bar{K}$ and $L_{*} /\left(h_{2}-h_{1}\right)$ are derived from the empirical functions of $F r_{1}$. Two values of $b$ are considered for $H_{c}^{\text {jet }}$ and $h_{2} / h_{1}: b=8$ (dashed line) and $b=12$ (dotted line). Also, the prediction from traditional hydraulics for horizontal flows is reported in continuous line for $H_{c}^{j e t}$.

traditional hydraulic equations (strictly valid for horizontal flows without any friction force considered). This explains the great success of traditional hydraulics for granular jumps in fast granular flows down steep slopes. However, the differences are more pronounced at low Froude numbers. Furthermore, changing $\tan \theta-\mu_{e}$ and/or the constant $b$ in the empirical relation for $L_{*} /\left(h_{2}-h_{1}\right)$ does significant change to the results at low Froude numbers. This highlights some uncertainty in defining the boundaries of the area of the phase-diagram at low values of $F r_{1}$ and $H / h_{1}$ (in which steady dead zones co-existing with flowing grains above can be formed) and a need for further investigations. In particular, the interaction between slow granular flows and walls should be investigated in more detail because it is entirely driven by the complex interplay between the hydrodynamic properties and frictional nature of the granular fluid. This slow dynamics is for instance crucial in geophysics and natural hazards mitigation, because it is relevant to situations where gravity 
mass movements interact with protection structures in the runout zones while the Froude number becomes relatively low.

\section{ACKNOWLEDGMENTS}

The author is grateful to the financial support by French National Research Agency through the project ANR-MOPERA and by the People Programme (Marie Curie Actions) of the European Unions Seventh Framework Programme under REA Grant Agreement No. 622899 (FP7-PEOPLE-2013-IOF, GRAINPACT).

[1] T. Chu, G. Hill, D. McClung, R. Ngun, and R. Sherkat, Canadian Geotechnical Journal 32, $285(1995)$

[2] D. M. McClung and A. I. Mears, Journal of Glaciology 41 (138), 359 (1995)

[3] S. Pudasaini, K. Hutter, S. Hsiau, S. Tai, Y. Wang, and R. Katzenbach, Physics of Fluids 19, $053302(2007)$

[4] S. P. Pudasaini and C. Kröner, Physical Review E 78, 041308 (2008)

[5] D. Mancarella and O. Hungr, Canadian Geotechnical Journal 47, 827 (2010)

[6] K. M. Hákonardóttir, T. Jóhannesson, F. Tiefenbacher, and M. Kern., A laboratory study of the retarding effect of breaking mounds in 3, 6 and $9 \mathrm{~m}$ long chutes., IMO Report 01007 (VEDURSTOFA ISLANDS, 2001)

[7] K. M. Hákonardóttir, A. Hogg, T. Jóhannesson, and G. G. Tómasson, Journal of Glaciology 49, $191(2003)$

[8] T. Faug, M. Naaim, D. Bertrand, P. Lachamp, and F. Naaim-Bouvet, Surveys in Geophysics 24, 555 (2003)

[9] T. Faug, M. Naaim, and F. Naaim-Bouvet, Cold Regions Science and Technology 39, 141 (2004)

[10] T. Faug, B. Chanut, M. Naaim, and B. Perrin, Annals of Glaciology 49, 77 (2008)

[11] T. Faug, P. Gauer, K. Lied, and M. Naaim, Journal of Geophysical Research 113, F03009 (2008)

[12] K. Platzer, S. Margreth, and P. Bartelt, in Snow engineering V, Bartelt, Adam, Christen, 
Sack and Sato (eds) Taylor and Francis Group, London. (2004)

[13] K. M. Hákonardóttir, A. Hogg, J. Batey, and A. Woods, Geophysical Research Letters 30, $2191(2003 \mathrm{a})$

[14] K. M. Hákonardóttir and A. Hogg, Physics of Fluids 17, 077101 (2005)

[15] M. Naaim, T. Faug, F. Naaim-Bouvet, and N. Eckert, Annals of Glaciology 51, 89 (2010)

[16] M. Chiou, Y. Wang, and K. Hutter, Acta Mechanica 175, 105 (2005)

[17] S. H. Chou, L. S. Lu, and S. S. Hsiau, Granular Matter 14, 719 (2012)

[18] B. Domnik, S. P. Pudasaini, R. Katzenbach, and S. A. Miller, Journal of Non-Newtonian Fluid Mechanics 201, 56 (2013)

[19] Y. C. Tai, J. M. N. T. Gray, K. Hutter, and S. Noelle, Annals of Glaciology 32, 281 (2001)

[20] J. M. N. T. Gray, Y. C. Tai, and S. Noelle, Journal of Fluids Mechanics , 161 (2003)

[21] T. Faug, M. Naaim, and F. Naaim-Bouvet, Annals of Glaciology 38, 135 (2004)

[22] M. Naaim, F. Naaim-Bouvet, T. Faug, and A. Bouchet, Cold Regions Science and Technology 39, $193(2004)$

[23] H. Teufelsbauer, Y. Wang, M. Chiou, and W. Wu, Granular Matter 11, 209 (2009)

[24] X. Cui and J. M. N. T. Gray, Journal of Fluid Mechanics 720, 314 (2013)

[25] K. Platzer, P. Bartelt, and C. Jaedicke, Cold Regions Science Technology 49, 11 (2007)

[26] K. M. Hákonardóttir, A. Hogg, T. Jóhannesson, M. Kern, and F. Tiefenbacher, Surveys in Geophysics 24, 543 (2003b)

[27] T. Faug, M. Naaim, and A. Fourriere, Cold Regions Science and Technology 49, 64 (2007)

[28] T. Faug, R. Beguin, and B. Chanut, Physical Review E 80, 021305 (2009)

[29] B. Chanut, T. Faug, and M. Naaim, Physical Review E 82, 041302 (2010)

[30] C.-Y. Kuo, L.-T. Sheng, S.-Y. Chiu, Y.-Z. Yang, Y.-C. Tai, and S.-S. Hsiau, Physics of Fluids 27, $013305(2015)$

[31] A. Albaba, S. Lambert, F. Nicot, and B. Chareyre, Granular Matter , 1 (2015)

[32] B. Domnik and S. P. Pudasaini, Journal of Non-Newtonian Fluid Mechanics 173, 72 (2012)

[33] S. P. Pudasaini and B. Domnik, Nonlinear Processes in Geophysics 16, 399 (2009)

[34] B. De Saint-Venant, Acad. Sci. Paris. Comptes rendus 73, 148 (1871)

[35] S. B. Savage and K. Hutter, Journal of Fluid Mechanics 199, 177 (1989)

[36] S. B. Savage and K. Hutter, Acta Mechanica 86, 201 (1991)

[37] P. Heinrich, A. Piatanesi, and H. Hebert, Geophysical Journal International 145, 97 (2001) 
[38] R. Iverson, Reviews of Geophysics 35, 245 (1997)

[39] D. Laigle and P. Coussot, Journal of Hydraulic Engineering - ASCE 123, 617 (1997)

[40] M. Naaim, S. Vial, and R. Couture, in Multiple Scale Analyses Coupled Phys. Syst.: SaintVenant Symp. Paris (Presse Ecole Nationale des Ponts et Chaussées, 1997)

[41] M. Naaim, T. Faug, and F. Naaim-Bouvet, Surveys in Geophysics , 569 (2003)

[42] O. Pouliquen and Y. Forterre, Journal of Fluid Mechanics 453, 133 (2002)

[43] L. Silbert, D. Ertas, G. S. Grest, T. C. Halsey, D. Levine, and S. J. Plimpton, Physical Review E 64, 051302 (2001)

[44] T. Weinhart, R. Hartkamp, A. R. Thornton, and S. Luding, Physics of Fluids 25, 070605 (2013)

[45] J. M. N. T. Gray, M. Wieland, and K. Hutter, Proceedings of the Royal Society of London A: Mathematical, Physical and Engineering Sciences 455, 1841 (1999)

[46] P. Johnson and R. Jackson, Journal of Fluid Mechanics 176, 67 (1987)

[47] P. Johnson, P. Nott, and R. Jackson, Journal of Fluid Mechanics 210, 501 (1990)

[48] M. Y. Louge, Physical Review E 67, 061303 (2003)

[49] D. Berzi, C. di Prisco, and D. Vescovi, Physical Review E 84, 031301 (2011)

[50] G. MiDi, European Physical Journal E 14, 341 (2004)

[51] P. Jop, O. Pouliquen, and Y. Forterre, Nature 441, 727 (2006)

[52] C. Johnson and J. Gray, Journal of Fluid Mechanics 675, 87 (2011)

[53] V. Chow, Open-Channels Hydraulics (McGraw-Hill, New York, 1959)

[54] H. L. Morrison and O. Richmond, Journal of Applied Mechanics 43, 49 (1976)

[55] S. B. Savage, Journal of Fluid Mechanics 92, 53 (1979)

[56] T. Faug, P. Childs, E. Wyburn, and I. Einav, Physics of Fluids 27, 073304 (2015)

[57] T. Faug, in AIP Conference Proceedings 1542, 642-645 (2013)

[58] J. M. N. T. Gray and K. Hutter, Continuum Mechanics and Thermodynamics 9, 341 (1997)

[59] Y. C. Tai and Y. Lin, Physics of Fluids 20, 123302 (2008)

[60] K. M. Hákonardóttir, The interaction between snow avalanches and dams, Phd thesis, School of Mathematics, University of Bristol (2004)

[61] T. Jóhannesson, K. M. Hákonardóttir, C. Harbitz, and U. Domaas, Background for the determination of dam height in the SATSIE dam design guidelines, Tech. Rep. (IMO Report 08003, 2008) 
[62] T. Jóhannesson, P. Gauer, D. Issler, and K. Lied, The design of avalanche protection dams. Recent practical and theoretical developments. (European Commission, contributions from M. Barbolini, U. Domaas, T. Faug, P. Gauer, K. M. Hákonardóttir, C.B. Harbitz, D. Issler, T. Jóhannesson, K. Lied, M. Naaim, F. Naaim-Bouvet and L. Rammer, 2009)

[63] C. Brennen, K. Sieck, and J. Paslaski, Powder Technology 35, 31 (1983)

[64] C. Campbell, C. Brennen, and R. Sabersky, Powder Technology 41, 77 (1985)

[65] A. Jaworski and T. Dyakowski, Experimental Thermal and Fluid Science 31, 877 (2007)

[66] T. Faug, P. Lachamp, and M. Naaim, Natural Hazards and Earth System Science 2, 187 (2002)

[67] K. M. Hákonardóttir, Retarding effects of braking mounds: Granular flows, Master's thesis, School of Mathematics, University of Bristol (2000)

[68] F. Naaim-Bouvet, M. Naaim, and T. Faug, Annals of Glaciology 38, 373 (2004)

[69] C. Yih, Fluid mechanics (West River Press, USA, 1979)

[70] P. Lachamp, T. Faug, M. Naaim, and D. Laigle, Natural Hazards and Earth System Science 2, $203(2002)$

[71] T. Faug, P. Caccamo, and B. Chanut, Geophysical Research Letters 39, L23401 (2012) 